\title{
Efficacy of circulating microRNA-130b and blood routine parameters in the early diagnosis of gastric cancer
}

\author{
JIANLIN CHEN $^{1 *}$, ZHAOHUI LIU $^{2 *}$, GAN GAO $^{3 *}$, YUANDONG MO ${ }^{4}$, HONGLING ZHOU $^{5}$, \\ WENJIE HUANG ${ }^{1}$, LIHUA WU ${ }^{6}$, XIAOLING HE ${ }^{7}$, JUNPING DING $^{8}$, CHANGJUN LUO $^{9}$, \\ HAIHUA LONG ${ }^{6}$, JINGRONG FENG ${ }^{8}$, YIFAN SUN ${ }^{1}$ and XIAOYONG GUAN ${ }^{10}$
}

\begin{abstract}
Departments of ${ }^{1}$ Clinical Laboratory and ${ }^{2}$ Anesthesia, Affiliated Liutie Central Hospital of Guangxi Medical University, Liuzhou, Guangxi Zhuang Autonomous Region 545007; ${ }^{3}$ Department of Clinical Laboratory, Liuzhou Maternity and Child Healthcare Hospital, Liuzhou, Guangxi Zhuang Autonomous Region 545001; Departments of ${ }^{4}$ General Surgery and

${ }^{5}$ Nursing, People's Hospital Rong'an County, Liuzhou, Guangxi Zhuang Autonomous Region 545400;

${ }^{6}$ Department of Digestive Internal Medicine, Affiliated Liutie Central Hospital of Guangxi Medical University,

Liuzhou, Guangxi Zhuang Autonomous Region 545007; ${ }^{7}$ Department of Clinical Laboratory,

People's Hospital Rong'an County, Liuzhou, Guangxi Zhuang Autonomous Region 545400;

Departments of ${ }^{8}$ General Surgery and ${ }^{9}$ Internal Medicine-Cardiovascular, Affiliated Liutie Central Hospital of

Guangxi Medical University, Liuzhou, Guangxi Zhuang Autonomous Region 545007;

${ }^{10}$ Department of Clinical Laboratory, The First Affiliated Hospital of Guangxi University of Science and

Technology, Liuzhou, Guangxi Zhuang Autonomous Region 545005, P.R. China
\end{abstract}

Received March 18, 2021; Accepted July 16, 2021

DOI: $10.3892 / \mathrm{ol} .2021 .12986$

\begin{abstract}
Patients with gastric cancer (GC) have a poor prognosis, which is mainly due to the low rate of early diagnosis. The present study aimed to evaluate whether circulating microRNA-130b (miR-130b) and blood routine parameters [neutrophil count (N\#), lymphocyte count (L\#), monocyte count $(\mathrm{M} \#)$, neutrophil percentage $(\mathrm{N} \%)$, lymphocyte percentage (L\%), monocyte percentage (M\%), hemoglobin $(\mathrm{Hb})$ level, hematocrit $(\mathrm{Hct})$, red blood cell distribution width (RDW), platelet count, platelet distribution width (PDW), mean platelet volume (MPV), MPV to platelet count ratio (MPV/PC), monocyte to lymphocyte ratio (MLR), neutrophil to lymphocyte ratio (NLR) and platelet to lymphocyte ratio (PLR)] are useful biomarkers for GC, early stage GC (EGC) and precancerous lesion (Pre) detection, and to identify more effective diagnostic models by combining circulating blood markers. Circulating levels
\end{abstract}

Correspondence to: Professor Xiaoyong Guan, Department of Clinical Laboratory, The First Affiliated Hospital of Guangxi University of Science and Technology, 124 Yuejin Road, Liubei, Liuzhou, Guangxi Zhuang Autonomous Region 545005, P.R. China E-mail: gxy6688@163.com

${ }^{*}$ Contributed equally

Key words: microRNA-130b, gastric cancer, diagnosis, blood routine parameters, biomarker of M\#, M\%, RDW-coefficient of variation (RDW-CV), MPV, PDW, MLR and NLR were significantly higher, and the levels of $\mathrm{Hb}$ and $\mathrm{L} \%$ were significantly lower in patients with GC and Pre compared with those in healthy controls (NCs) (all $\mathrm{P}<0.05$ ). The $\mathrm{N} \#, \mathrm{~N} \%$ and PLR in patients with GC were significantly higher and the Hct was significantly lower than those in the NCs (all $\mathrm{P}<0.05)$. The values of MPV/PC were significantly higher in the Pre cohort compared with those in the NCs. The area under the curve (AUC) of the receiver operating characteristic curve of potential biomarkers for GC was 0.634-0.887 individually, and this increased to 0.978 in the combination model of miR-130b-PDW-MLR-Hb. Additionally, the values for RDW-CV, PLR, NLR, N\# and $\mathrm{N} \%$ were positively correlated with cancer stage, while the values for MPV, L\#, L\%, Hb and Hct were negatively correlated with cancer stage. Furthermore, the circulating levels of miRNA-130b, and the values for NLR, RDW-CV, PDW, M\%, red blood cell count, Hct, Hb and MLR differed between the EGC and NC groups. The AUC values of these biomarkers were 0.6491-0.911 individually in the diagnosis of EGC, and these increased to 0.960 in combination. In addition, the AUC values for miR-130b, RDW-CV, MPV/PC ratio, MLR, NLR, PDW, $\mathrm{L} \%, \mathrm{M} \%, \mathrm{M} \#$ and $\mathrm{Hb}$ in the diagnosis of Pre were 0.638-0.811 individually. The dual-model of miR-130b-PDW manifested the largest AUC of 0.896 in the diagnosis of Pre, and the sensitivity and accuracy were increased when miR-130b and PDW were combined. All these results suggested that circulating miR-130b and blood routine parameters might be potential biomarkers, and combinations of measurements of these biomarkers may improve the GC, EGC and Pre diagnostic accuracy. 


\section{Introduction}

It has been reported that gastric cancer (GC) is the second leading cause of cancer-associated mortality worldwide (1). Although extensive studies have been conducted, few risk factors have been confirmed and there are no effective biomarkers or screening tools for early detection in asymptomatic individuals $(2,3)$. Conventional tumor markers, such as CEA, CA72-4 and CA19-9, are useful only for identifying cases of advanced GC and monitoring GC recurrence (3). However, these serum markers lack sufficient sensitivity and specificity (4). Currently, endoscopy and the barium meal test are the main methods used for the clinical diagnosis of GC. However, due to their invasiveness, patient compliance with this procedure is poor (1). Chronic inflammation is not an important cause of GC; however, it is considered to be involved in the pathogenesis of $25 \%$ of all cancer cases worldwide (5). Blood routine parameters have been introduced as biomarkers for the diagnosis of numerous cancer-related diseases associated with inflammatory processes (6-10). However, to the best of our knowledge, the longitudinal changes of these parameters in different stages of GC progression have not been studied comprehensively at present.

MicroRNAs (miRNAs/miRs) are members of the endogenous, non-coding single-stranded RNA family, and are released from tissues to extracellular biofluids after receiving inflammatory stimulation (11). Specific miRNAs, which have carcinogenic or tumor-suppressive activities, may be mediators for inflammation to induce carcinogenesis (12), and have been detected in numerous types of cancer, including breast cancer, hepatocellular carcinoma and other cancer types (13-15). Numerous studies have demonstrated that non-invasive circulating miRNA recognition is valuable and useful in diseases, including GC (16,17). However, these studies have not included patients with early stage GC (EGC) or patients with a precancerous lesion (Pre), although an ideal non-invasive marker should be able to identify both of these stages.

A recent study has reported that the combination of miR-650 and CA211 can distinguish between benign and malignant GC (18). Therefore, we hypothesized that the combined assessment of miRNAs and blood routine parameters may reveal novel insights for the diagnosis of GC. The present study examined the circulating levels of miR-130b and blood routine parameters in 90 patients with GC, 90 patients with a Pre and 45 healthy individuals. Subsequently, statistical analysis was performed to compare the diagnostic value of these markers for GC. The present study indicated that plasma miR-130b and complete blood count parameters might be promising non-invasive biomarkers for the early detection of GC, and combined utilization of these markers could improve the efficacy of the early diagnosis of GC.

\section{Materials and methods}

Patients and healthy control characteristics. The present study included 90 patients with GC, 90 patients with Pres and 45 healthy controls (NCs) who received treatment at the Affiliated Liutie Central Hospital of Guangxi Medical University (Liuzhou, China) between January 2014 and March 2019. Patients with GC and Pres were selected according to gastroscopy combined with histological examination and the blood samples were collected prior to any surgery and therapy. Patients who had received radiation therapy or chemotherapy were excluded from the study.

The histological type and tumor stage were identified according to the Union of International Cancer Control TNM system, 7th edition (19). The histology of all patients was assessed according to World Health Organization criteria (20). NCs had no history of diabetes, heart disease, hypertension or cancer, and attended the Affiliated Liutie Central Hospital of Guangxi Medical University for routine health checks. Approval for the study was obtained from the Ethics Committee of the Affiliated Liutie Central Hospital of Guangxi Medical University, and written informed consent was issued by all study participants.

Plasma preparation and miRNA extraction. Peripheral blood samples were drawn using EDTA anticoagulative tubes prior to surgical treatment. Cell-free plasma samples were centrifuged at $1,520 \mathrm{x} \mathrm{g}$ for $5 \mathrm{~min}$ to prevent contamination by cellular nucleic acids and stored at $-80^{\circ} \mathrm{C}$ until miRNA extraction or kept at $-20^{\circ} \mathrm{C}$ for conventional tumor marker determination.

$R N A$ isolation and reverse transcription-quantitative PCR (RT-qPCR) assay. Total RNA was isolated from $200 \mu$ l plasma using a Blood (Serum/Plasma) MicroRNA Extraction and Purification kit (spin column) (Novland Co., Ltd.; http://www. novland.com.cn/). The concentration of total RNAs was quantified using a NanoQ micro-volume Spectrophotometer (CapitalBio Technology, Inc.). Circulating miRNA-130b expression was determined by RT-qPCR using a One Step qRT-PCR Kit (with Taqman probes; catalog no. LK-0106B) with $2 \mu \mathrm{l}$ initial template, while miR-16 served as an internal control (21). Thermocycling conditions were $45^{\circ} \mathrm{C}$ for $30 \mathrm{~min}$ for reverse transcription, then $94^{\circ} \mathrm{C}$ for $2 \mathrm{~min}$, followed by 40 cycles of $94^{\circ} \mathrm{C}$ for $15 \mathrm{sec}, 55^{\circ} \mathrm{C}$ for $45 \mathrm{sec}$ and $72^{\circ} \mathrm{C}$ for 60 sec. RT-qPCR was performed on an ABI 7500 Real-Time PCR System (Applied Biosystems; Thermo Fisher Scientific, Inc.). The primer sequences for PCR were as follows: miR-130b forward, 5'-GACACUCUUUCCCUGUUGCAC UACU-3' and reverse, 5'-CAGTGCGTGTCGTGGAGT-3'; and reference miRNA (hsa-miR-16) forward, 5'-GTCGTA TCCAGTGCAGGGTCCGAGTCGCACTGGATACGACCG CCAA-3' and reverse, 5'-GTATCCAGTGCAGGGTCCGAG GT-3'. Expression levels of target miRNAs were calculated using the quantification cycle $(\mathrm{Cq})$ values with SDS 2.0 software (Applied Biosystems; Thermo Fisher Scientific, Inc.). The relative expression levels of $\mathrm{miR}-130 \mathrm{~b}$ were calculated using the $2^{-\Delta \Delta \mathrm{Cq}}$ method (22), in which $\Delta \mathrm{Cq}=\mathrm{Cq}(\mathrm{miR}-130 \mathrm{~b})-\mathrm{Cq}(\mathrm{miR}-16)$.

Blood parameter testing. Hematological parameters, including red blood cell count $(\mathrm{R} \#)$, hemoglobin $(\mathrm{Hb})$, hematocrit $(\mathrm{Hct})$, white blood cell count (WBC), neutrophil count (N\#), lymphocyte percentage (L\#), monocyte count (M\#), platelet count (PLT) and mean platelet volume (MPV), were measured before radical surgery using the automatic blood analyzer 800i (Sysmex Corporation) according to the manufacturer's protocols. Conventional tumor markers, including CA125, CA211 and CA50, were detected using a Roche cobas E170 analyzer (Roche Diagnostics). 
A

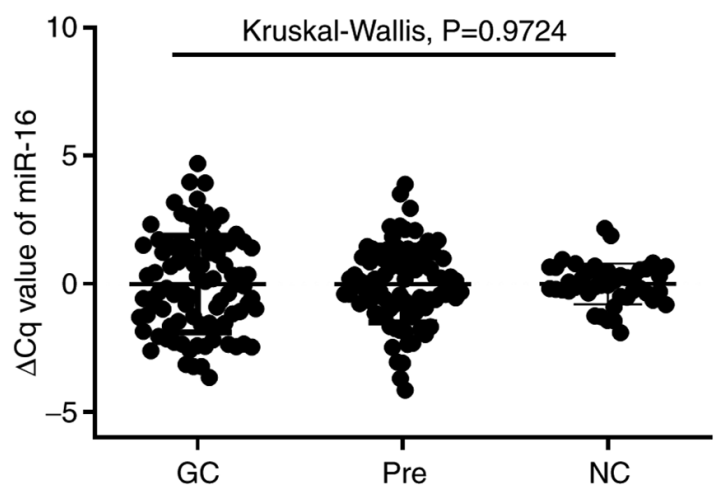

B

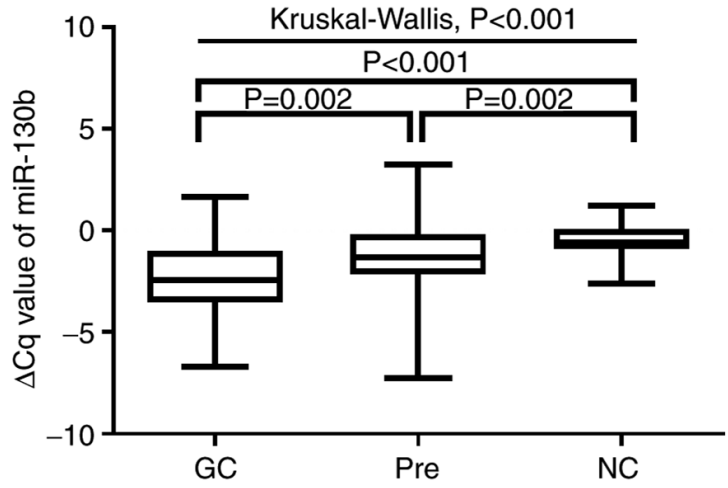

Figure 1. miR-130b expression in different groups. (A) Differential expression levels of reference miR-16 (P>0.05). (B) $\Delta$ Cq values of miR-130b in different groups $(\mathrm{P}<0.05)$. The $\Delta \mathrm{Cq}$ values denote the normalized $\mathrm{Cq}$ value by subtracting the $\mathrm{Cq}$ value of miR-16 from that of miR-130b. A lower $\Delta \mathrm{Cq}$ value indicated a higher level of miR-130b expression. The Kruskal-Wallis $\mathrm{H}$ test was used to compare variables between three groups, and the Bonferroni method was used to correct for post hoc pairwise comparisons of significance levels. GC, gastric cancer; miR, microRNA; NC, healthy control; Pre, precancerous lesions.

Statistical analysis. All experiments were repeated three times independently, and the data are expressed as the mean \pm SD. Differences in relevant indicators between two groups were compared using an unpaired t-test, or the Mann-Whitney U test when the conditions for the Student's t-test were not satisfied. Differences in relevant indicators among three groups were analyzed using one-way ANOVA, or Kruskal-Wallis H test when the conditions for one-way ANOVA were not satisfied. Tukey's honest significant difference test was used as a post hoc test after one-way ANOVA, and the Bonferroni method was used to correct for post hoc pairwise comparisons of significance levels after the Kruskal-Wallis $\mathrm{H}$ test. Differences in sex distribution among the three groups were analyzed using the $\chi^{2}$ test. All statistical analyses and generation of images were performed using SPSS 20.0 software (IBM Corp.) or GraphPad Prism 8.0 Software (GraphPad Software, Inc.). Correlations between circulating biomarkers in GC were analyzed using Pearson's correlation. Correlations between blood biochemical indexes and cancer stage in GC were analyzed by Spearman's correlation. Sensitivity and specificity were defined by receiver operating characteristic (ROC) curves, and differences in the area under the curve (AUC) and diagnostic accuracy parameters, including sensitivity, specificity, negative likelihood ratio ( $\left.\mathrm{LR}^{-}\right)$, positive likelihood ratio $\left(\mathrm{LR}^{+}\right)$and diagnostic odds ratio (DOR) were detected using MedCalc version 19.5.3 (MedCalc Software, Ltd.). For all analyses, $\mathrm{P}<0.05$ was considered to indicate a statistically significant difference.

\section{Results}

Expression levels of miR-130b, complete blood count parameters and conventional tumor markers in GC, Pre and NCs. A total of 90 patients with GC, 90 patients with Pres and 45 NCs were included in the present study. Plasma miR-130b expression was examined by RT-qPCR. As shown in Fig. 1A, no significant differences in miR-16 expression were observed among the three groups $(\mathrm{P}=0.9724)$. As shown in Fig. 1B, plasma miRNA-130b levels in patients with GC and Pres were significantly higher than those of NCs (all $\mathrm{P}<0.05)$. Furthermore, the plasma miRNA-130b levels in GC were significantly higher than those in the Pre group $(\mathrm{P}=0.002)$. As shown in Table $\mathrm{I}$,

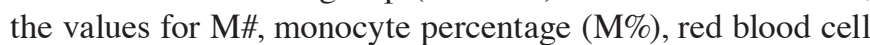
distribution width-coefficient of variation (RDW-CV), MPV, monocyte to lymphocyte count ratio (MLR), neutrophil to lymphocyte ratio (NLR), CA211 and CA50 in patients with GC and Pres were significantly higher than those in NCs (all $\mathrm{P}<0.05)$. Notably, patients with GC and Pres had significantly lower $\mathrm{Hb}, \mathrm{L} \%$ and platelet distribution width (PDW) than NCs (GC vs. NC, $\mathrm{P}<0.05$; Pre vs. NC, $\mathrm{P}<0.05$; Table I). The values for N\#, neutrophil percentage (N\%), platelet to lymphocyte ratio (PLR) and CA125 in patients with GC were significantly higher, while Hct was significantly lower, compared with those in the normal group (all $\mathrm{P}<0.05$ ). Furthermore, the MPV to platelet count ratio (MPV/PC) was significantly higher in the Pre cohort $(0.04 \pm 0.02)$ compared with $\mathrm{NCs}(0.03 \pm 0.01$; $\mathrm{P}=0.004)$. In addition, the values for WBC and PLT did not differ significantly among the three groups.

Diagnostic values of tumor markers for GC based on AUC analysis. To further assess the diagnostic value of potential diagnostic biomarkers for GC, ROC analyses were subsequently performed, and the key indicators, including sensitivity, specificity, positive predictive value, negative predictive value, cut-off value and AUC, were measured as shown in Fig. 2 and Table II. ROC analyses suggested that the AUC was calculated as 0.887 for miR-130b, 0.828 for $\mathrm{Hb}, 0.764$ for Hct, 0.763 for RDW-CV, 0.667 for MPV, 0.757 for NLR, 0.814 for MLR, 0.712 for PLR, 0.840 for PDW, 0.709 for $\mathrm{N} \%, 0.715$ for $\mathrm{M} \%, 0.782$ for $\mathrm{L} \%, 0.634$ for $\mathrm{N} \#, 0.680$ for $\mathrm{M \#}$ and 0.719 for lymphocyte count (L\#). At a cut-off value of 0.18 , circulating miR-130b exhibited the highest diagnostic accuracy and sensitivity compared with any other parameters, highlighting its potential as an effective biomarker for GC. Our previous study also assayed CA211 and CA50 levels in the same plasma samples, and at cut-off values of $2.2 \mathrm{ng} / \mathrm{ml}$ for CA211 and $7.96 \mathrm{ng} / \mathrm{ml}$ for CA50, the sensitivity and specificity values were 80.65 and $95.65 \%$, and 55.84 and $85.29 \%$, respectively (23).

Integrative diagnosis model for detecting GC. To further assess the effect of circulating biomarkers, an integrative diagnosis 
Table I. Blood biochemical examination results of subjects.

\begin{tabular}{|c|c|c|c|c|c|c|c|c|c|c|}
\hline \multirow[b]{2}{*}{ Variables } & \multicolumn{2}{|r|}{ GC } & \multicolumn{2}{|r|}{ Pre } & \multicolumn{2}{|r|}{$\mathrm{NC}$} & \multirow[b]{2}{*}{ P-value ${ }^{a}$} & \multirow{2}{*}{$\begin{array}{c}\text { GC vs. } \\
\text { Pre } \\
\text { P-value }\end{array}$} & \multirow{2}{*}{$\begin{array}{c}\text { GC vs. } \\
\text { NC } \\
\text { P-value }\end{array}$} & \multirow{2}{*}{$\begin{array}{c}\text { Pre vs. } \\
\text { NC } \\
\text { P-value }\end{array}$} \\
\hline & $\begin{array}{c}\text { No. of } \\
\text { patients }\end{array}$ & Mean \pm SD & $\begin{array}{c}\text { No. of } \\
\text { patients }\end{array}$ & Mean \pm SD & $\begin{array}{c}\text { No. of } \\
\text { patients }\end{array}$ & Mean \pm SD & & & & \\
\hline WBC, $10^{9} / 1$ & 89 & $6.41 \pm 2.98$ & 76 & $6.25 \pm 2.68$ & 45 & $6.01 \pm 1.28$ & 0.2846 & - & - & - \\
\hline $\mathrm{N} \#, 10^{9} / 1$ & 89 & $4.55 \pm 2.86$ & 76 & $3.84 \pm 2.61$ & 45 & $3.61 \pm 0.98$ & 0.0445 & $>0.999$ & 0.039 & 0.228 \\
\hline $\mathrm{L \#}, 10^{9} / 1$ & 89 & $1.34 \pm 0.66$ & 76 & $1.66 \pm 0.76$ & 45 & $1.93 \pm 0.73$ & $<0.0001$ & 0.002 & 0.001 & 0.169 \\
\hline $\mathrm{M} \#, 10^{9} / 1$ & 89 & $0.31 \pm 0.21$ & 76 & $0.31 \pm 0.17$ & 45 & $0.24 \pm 0.4$ & 0.0018 & $>0.999$ & 0.002 & 0.015 \\
\hline N\% & 89 & $67.81 \pm 14.75$ & 76 & $63.45 \pm 12.65$ & 45 & $61.23 \pm 7.99$ & 0.0003 & 0.052 & 0.001 & 0.260 \\
\hline $\mathrm{L} \%$ & 89 & $22.74 \pm 11.4$ & 76 & $29.31 \pm 11.63$ & 45 & $31.90 \pm 7.57$ & $<0.0001$ & 0.025 & 0.016 & 0.001 \\
\hline M\% & 89 & $5.31 \pm 3.75$ & 76 & $5.05 \pm 2.17$ & 45 & $3.71 \pm 1.35$ & 0.0003 & 0.237 & 0.001 & 0.023 \\
\hline $\mathrm{Hb}, \mathrm{g} / \mathrm{l}$ & 89 & $116.10 \pm 32.22$ & 76 & $128.04 \pm 21.67$ & 45 & $138.46 \pm 16.05$ & $<0.0001$ & 0.001 & 0.01 & 0.001 \\
\hline Hct, $\%$ & 89 & $35.70 \pm 9.07$ & 76 & $39.21 \pm 6.18$ & 45 & $40.43 \pm 3.92$ & $<0.0001$ & 0.001 & 0.001 & $>0.999$ \\
\hline RDW-CV, \% & 89 & $14.01 \pm 3.24$ & 76 & $12.75 \pm 1.92$ & 45 & $12.31 \pm 1.40$ & $<0.0001$ & 0.001 & 0.001 & 0.048 \\
\hline PLT, $10^{12} / 1$ & 89 & $256.00 \pm 111.43$ & 76 & $231.50 \pm 63.38$ & 45 & $248.00 \pm 54.68$ & 0.0704 & - & - & - \\
\hline PDW, \% & 89 & $11.65 \pm 2.77$ & 76 & $11.91 \pm 2.51$ & 45 & $15.90 \pm 0.38$ & $<0.0001$ & $>0.999$ & 0.001 & 0.001 \\
\hline MPV, fl & 89 & $9.62 \pm 1.20$ & 76 & $9.91 \pm 1.14$ & 45 & $8.90 \pm 1.39$ & $<0.0001^{\mathrm{b}}$ & 0.046 & 0.003 & 0.001 \\
\hline $\mathrm{MPV} / \mathrm{PC}$ & 89 & $0.04 \pm 0.04$ & 76 & $0.04 \pm 0.02$ & 45 & $0.03 \pm 0.01$ & 0.0047 & 0.105 & 0.428 & 0.004 \\
\hline MLR & 89 & $0.24 \pm 0.41$ & 76 & $0.17 \pm 0.18$ & 45 & $0.11 \pm 0.98$ & $<0.0001$ & 0.006 & 0.001 & 0.003 \\
\hline NLR & 89 & $2.94 \pm 9.33$ & 76 & $2.20 \pm 6.09$ & 45 & $1.96 \pm 0.63$ & $<0.0001$ & 0.001 & 0.001 & 0.001 \\
\hline PLR & 89 & $198.05 \pm 286.52$ & 76 & $131.03 \pm 112.07$ & 45 & $134.36 \pm 76.12$ & $<0.0001$ & 0.001 & 0.001 & 0.031 \\
\hline $\mathrm{CA} 125, \mathrm{U} / \mathrm{ml}$ & 68 & $59.22 \pm 92.72$ & 53 & $12.36 \pm 7.78$ & 45 & $11.07 \pm 4.05$ & 0.0078 & 0.024 & 0.028 & $>0.999$ \\
\hline $\mathrm{CA} 211, \mathrm{ng} / \mathrm{ml}$ & 40 & $16.15 \pm 44.84$ & 62 & $2.95 \pm 1.38$ & 45 & $2.09 \pm 0.48$ & $<0.0001$ & 0.045 & 0.001 & 0.001 \\
\hline CA50, U/ml & 48 & $38.01 \pm 99.02$ & 77 & $8.18 \pm 5.97$ & 34 & $4.91 \pm 4.46$ & $<0.0001$ & 0.001 & 0.001 & 0.001 \\
\hline
\end{tabular}

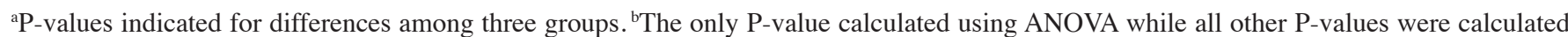
using Kruskal-Wallis. $\mathrm{P}<0.05$ was considered to indicate a statistically significant difference. Some data are missing due to retrospective data collection. GC, gastric cancer; Pre, precancerous lesions; NC, healthy control; WBC, white blood cell count; N\#, neutrophil count; L\#, lymphocyte count; M\#, monocyte count; N\%, neutrophil percentage; L\%, lymphocyte percentage; M\%, monocyte percentage; Hb, hemoglobin; Hct, hematocrit; RDW-CV, red blood cell distribution width-coefficient of variation; PLT, platelet count; PDW, platelet distribution width; MPV, mean platelet volume; MPV/PC, mean platelet volume/platelet count ratio; MLR, monocyte to lymphocyte count ratio; NLR, neutrophil to lymphocyte ratio; PLR, platelet to lymphocyte count ratio.

model was generated for discriminating GC. The circulating markers with AUC >0.80 (miR-130b, Hb, MLR and PDW) were further considered for combinations. For the two-dimensional models, PDW-Hb yielded the greatest AUC (0.945), followed by miR-130b-Hb (0.937), miR-130b-PDW (0.922), miR-130b-MLR (0.892), PDW-MLR (0.843) and MLR-Hb (0.835). The AUC values of the dual-models miR-130b-PDW, miR-130b-Hb and PDW-Hb were all greater than those of any of the one-dimensional models. However, there was no statistically significant difference in AUC between the PDW-MLR and MLR-Hb dual-models compared with the corresponding one-dimensional models. In addition, the AUC of the dual-model miR-130b-MLR was significantly different from that of the miR-130b one-dimensional model, while miR-130b-MLR was not statistically significantly different compared with the MLR one-dimensional model. For the miR-130b-PDW-MLR three-dimensional model, the AUC (0.955) was greater than those of the miR-130b-MLR or the PDW-MLR two-dimensional models (all $\mathrm{P}<0.05$; Table III). Similarly, the AUC (0.976) of the three-dimensional model miR-130b-PDW-Hb was greater than those of the
miR-130b-Hb or the PDW-Hb two-dimensional models. The AUC (0.942) of the miR-130b-MLR-Hb three-dimensional model was greater than those of the miR-130b-MLR or the MLR-Hb two-dimensional models. For the PDW-MLR-Hb three-dimensional model, the AUC (0.947) was greater than those of the PDW-MLR or MLR-Hb two-dimensional models. Furthermore, for the four-dimensional model miR-130b-PDW-MLR-Hb, the AUC (0.978) was greater than those of the three-dimensional models miR-130b-MLR-Hb and PDW-MLR-Hb (all $\mathrm{P}<0.05$; Table III). These results indicated that the combination of different biomarkers can improve the diagnostic efficiency to some extent.

Diagnostic value of four-dimensional biomarkers in GC. The corresponding diagnostic accuracy parameters, including sensitivity, specificity, $\mathrm{LR}^{-}, \mathrm{LR}^{+}$and DOR, are shown in Table IV. miR-130b exhibited a sensitivity and specificity of 91.11 and $71.11 \%$, PDW had a sensitivity and specificity of 70.45 and $97.78 \%$, MLR exhibited a sensitivity and specificity of 80.90 and $75.56 \%$, and $\mathrm{Hb}$ had a sensitivity and specificity of 68.54 and $84.44 \%$, respectively. miR-130b-PDW 

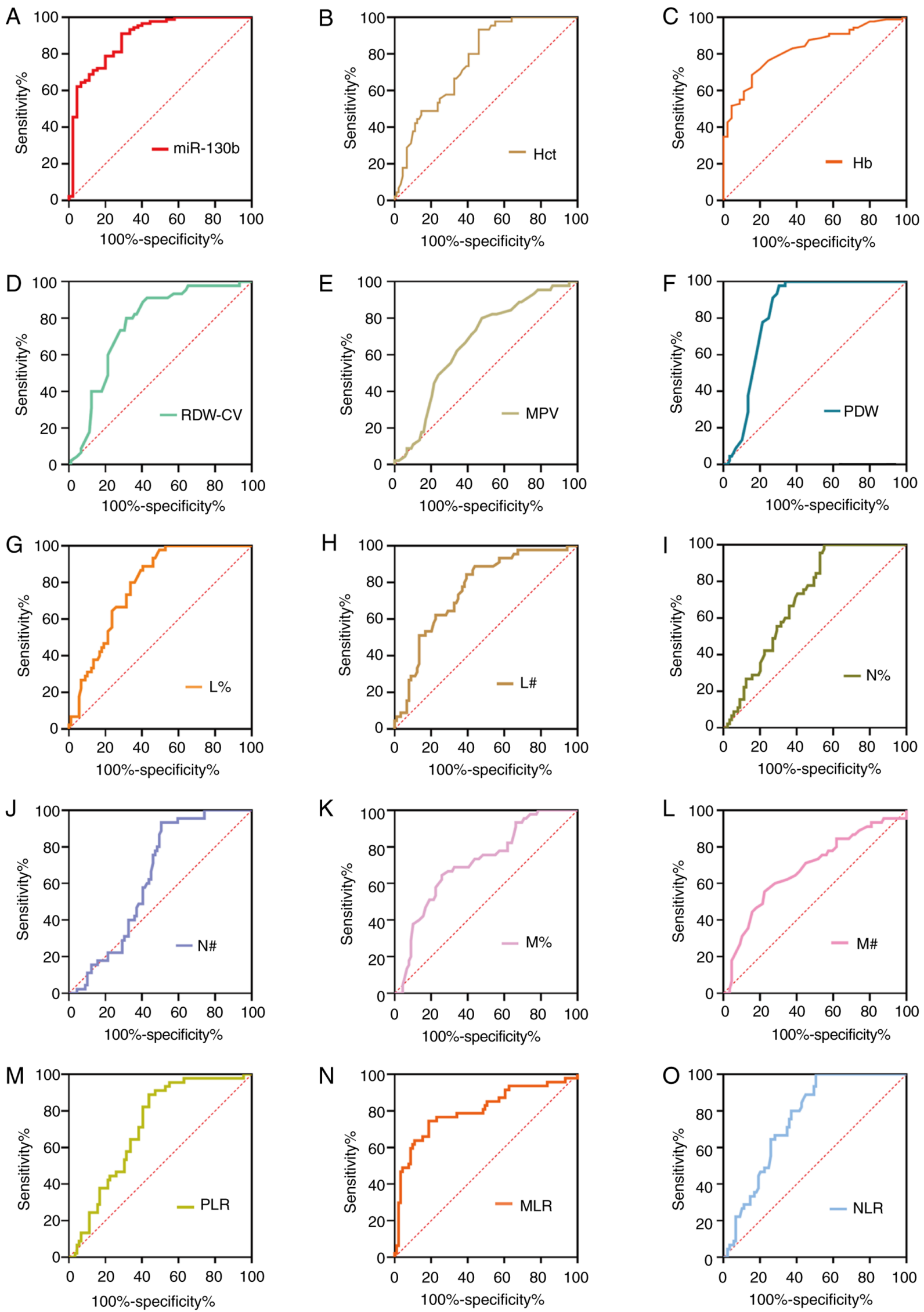

Figure 2. ROC curve analyses of tumor markers for gastric cancer. ROC curve analysis of biomarkers (A) miR-130b, (B) Hct, (C) Hb, (D) RDW-CV, (E) MPV, (F) PDW, (G) L\%, (H) L\#, (I) N\%, (J) N\#, (K) M\%, (L) M\#, (M) PLR, (N) MLR and (O) NLR for discrimination between gastric cancer and healthy controls. Hb, hemoglobin; Hct, hematocrit; L\%, lymphocyte percentage; L\#, lymphocyte count; miR-130b, microRNA-130b; MLR, monocyte to lymphocyte ratio; MPV, mean platelet volume; M\%, monocyte percentage; M\#, monocyte count; N\%, neutrophil percentage; N\#, neutrophil count; NLR, neutrophil to lymphocyte ratio; PDW, platelet distribution width; PLR, platelet to lymphocyte ratio; RDW-CV, red blood cell distribution width-coefficient of variation; ROC, receiver operating characteristic. 
Table II. Diagnostic value of different indicators for gastric cancer.

\begin{tabular}{|c|c|c|c|c|c|c|c|c|c|}
\hline Variable & $\begin{array}{c}\text { AUC } \\
(95 \% \text { CI })\end{array}$ & $\begin{array}{l}\text { Cut-off } \\
\text { index } \mathrm{J}\end{array}$ & Youden & Sensitivity, $\%$ & Specificity, \% & Accuracy, $\%$ & PPV, \% & NPV, $\%$ & P-value \\
\hline miR-130b & $0.887(0.821-0.935)$ & 0.18 & 0.6222 & 91.11 & 71.11 & 84.44 & 14.00 & 35.99 & 0.001 \\
\hline $\mathrm{Hb}, \mathrm{g} / \mathrm{l}$ & $0.828(0.753-0.888)$ & 127.00 & 0.5298 & 68.54 & 84.44 & 73.87 & 8.00 & 39.35 & 0.001 \\
\hline Hct, \% & $0.764(0.682-0.833)$ & 36.11 & 0.4727 & 53.93 & 93.33 & 67.16 & 4.00 & 43.02 & 0.001 \\
\hline RDW-CV, \% & $0.763(0.681-0.832)$ & 12.80 & 0.4854 & 68.54 & 80.00 & 72.39 & 10.00 & 37.28 & 0.001 \\
\hline MPV, fl & $0.667(0.580-0.747)$ & 9.50 & 0.3227 & 52.27 & 80.00 & 61.65 & 10.00 & 36.85 & 0.001 \\
\hline NLR & $0.757(0.675-0.827)$ & 3.13 & 0.4944 & 49.44 & 100.00 & 66.42 & 1.00 & 46.00 & 0.001 \\
\hline MLR & $0.814(0.738-0.876)$ & 0.15 & 0.5645 & 80.90 & 75.56 & 79.10 & 11.99 & 36.00 & 0.001 \\
\hline PLR & $0.712(0.628-0.787)$ & 188.98 & 0.4507 & 56.18 & 88.89 & 67.16 & 5.99 & 41.02 & 0.001 \\
\hline PDW, \% & $0.840(0.766-0.898)$ & 15.31 & 0.6823 & 70.45 & 97.78 & 79.70 & 1.99 & 45.69 & 0.001 \\
\hline $\mathrm{N} \%$ & $0.709(0.624-0.784)$ & 70.40 & 0.4494 & 44.94 & 100.00 & 63.43 & 1.00 & 45.91 & 0.001 \\
\hline $\mathrm{M} \%$ & $0.715(0.630-0.789)$ & 4.21 & 0.3860 & 74.16 & 64.44 & 70.90 & 17.00 & 30.25 & 0.001 \\
\hline $\mathrm{L} \%$ & $0.782(0.703-0.849)$ & 25.22 & 0.4844 & 59.55 & 88.89 & 69.40 & 5.99 & 41.11 & 0.001 \\
\hline $\mathrm{N} \#, 10^{9} / 1$ & $0.634(0.547-0.716)$ & 4.63 & 0.4277 & 49.44 & 93.33 & 64.18 & 4.00 & 42.93 & 0.0044 \\
\hline $\mathrm{M} \#, 10^{9} / 1$ & $0.680(0.594-0.758)$ & 0.24 & 0.3308 & 77.53 & 55.56 & 70.15 & 20.99 & 26.25 & 0.0004 \\
\hline $\mathrm{L \#}, 10^{9} / 1$ & $0.719(0.635-0.793)$ & 1.46 & 0.4067 & 60.67 & 80.00 & 67.16 & 10.00 & 37.02 & 0.001 \\
\hline
\end{tabular}

Sensitivity, specificity, PPV, NPV, accuracy and Youden index J were calculated based on the numbers of positive and negative cases. AUC, area under the curve; 95\% CI, 95\% confidence interval; PPV, positive predictive value; NPV, negative predictive value; miR-130b, microRNA-130b; Hb, hemoglobin; Hct, hematocrit; RDW-CV, red blood cell distribution width-coefficient of variation; MPV, mean platelet volume; NLR, neutrophil to lymphocyte ratio; MLR, monocyte to lymphocyte count ratio; PLR, platelet to lymphocyte count ratio; PDW, platelet distribution width; N\%, neutrophil percentage; M\%, monocyte percentage; L\%, lymphocyte percentage; N\#, neutrophil count; M\#, monocyte count; L\#, lymphocyte count.

had a sensitivity of $82.02 \%$ and a specificity of $88.89 \%$, miR-130b-MLR had a sensitivity of $75.28 \%$ and a specificity of $86.67 \%$, miR-130b-Hb had a sensitivity of $89.89 \%$ and a specificity of $86.67 \%$, PDW-MLR had a sensitivity of $71.26 \%$ and specificity of $97.78 \%$, PDW-Hb had a sensitivity of $89.66 \%$ and a specificity of $88.89 \%$, and MLR-Hb had a sensitivity of $73.03 \%$ and a specificity of $84.44 \%$. The three-dimensional model of miR-130b-RDW-MLR yielded a sensitivity of $94.25 \%$ and a specificity of $91.11 \%$ at the optimal cut-off point, miR-130b-PDW-Hb yielded a sensitivity of $90.80 \%$ and specificity of $97.78 \%$ at the optimal cut-off point of 0.77 as the optimal cut-off point, miR-130b-MLR-Hb yielded a sensitivity of $91.01 \%$ and a specificity of $86.67 \%$ at the 0.52 as the optimal cut-off point, and PDW-MLR-Hb yielded a sensitivity of $89.66 \%$ and a specificity of $91.11 \%$ at 0.55 as the optimal cut-off point. Furthermore, the four-dimensional model miR-130b-PDW-MLR-Hb had a sensitivity of $91.95 \%$ and a specificity of $97.78 \%$ at 0.76 as the optimal cut-off point. Furthermore, the combination of miR-130b-PDW-MLR-Hb yielded the largest accuracy, $\mathrm{LR}^{+}$and $\mathrm{DOR}$ values.

Association between the levels of circulating biomarkers and the risk of $G C$. To further determine whether the circulating marker levels for GC were associated with the presence of GC, logistic regression analysis was performed with the biomarkers as dependent variables (Table V). The crude odds ratio was obtained from logistic regression analysis, and the adjusted odds ratio was evaluated by adjusting for age and sex. The results demonstrated that the increase of miR-130b, RDW-CV, MPV, NLR, PLR, N\% and M\% was positively associated with the presence of GC (all $\mathrm{P}<0.05$; Table V). The levels of PDW, $\mathrm{Hb}, \mathrm{Hct}, \mathrm{L} \%$ and $\mathrm{L \#}$ were negatively associated with the risk of GC (all P<0.05), while the levels of MLR, N\# and M\# were not associated with the incidence of GC (all P $>0.05$; Table V).

Association between circulating biomarkers and conventional GC markers. Correlation analysis was performed as shown in Fig. 3. Correlation analysis demonstrated that the value of RDW-CV was positively associated with the concentrations of CA50 $(\mathrm{R}=0.2424 ; \mathrm{P}=0.0337$; Fig. 3A) and CA125 ( $\mathrm{R}=0.3426$; $\mathrm{P}=0.0042$; Fig. 3B). Pearson's correlation analysis indicated that PLR $(\mathrm{R}=0.2568$; $\mathrm{P}=0.0346$; Fig. 3C) and N\% (R=0.2886; $\mathrm{P}=0.0170$; Fig. 3D) were positively associated with the CA125 concentration, and L\% (R, -0.367; P=0.0022; Fig. 3O) was negatively correlated with the CA125 concentration. Furthermore, the analysis demonstrated that MPV, N\#, NLC and M\# were positively associated with the CA211 concentration (all $\mathrm{P}<0.05$; Fig. 3E-H). Particularly, $\mathrm{Hb}$ and Hct were negatively correlated with the concentration of CA125, CA211 and CA50 (all P<0.05; Fig. 3I-N). However, miR-130b, MLR and PDW were not correlated with CA125, CA50 or CA211 (all $\mathrm{P}>0.05$; data not shown).

Levels of circulating biomarkers are associated with severity of $G C$. Correlations between cancer stage and RDW-CV, PLR, NLR, MPV, PDW, N\#, M\%, miR-130b, N\%, M\#, L\#, $\mathrm{L} \%, \mathrm{Hb}, \mathrm{Hct}$ and MLR in patients with GC are shown in Fig. 4. Correlation analysis revealed that RDW-CV, PLR, NLR, N\# and N\% were positively correlated with cancer 
Table III. Receiver operating characteristic analysis of circulating biomarkers individually and combined for gastric cancer detection.

\begin{tabular}{|c|c|c|c|c|c|c|}
\hline Models & AUC $(95 \% \mathrm{CI})$ & Cut-off & $\begin{array}{c}\text { Sensitivity, } \\
\%\end{array}$ & $\begin{array}{c}\text { Specificity, } \\
\%\end{array}$ & $\begin{array}{l}\text { Youden } \\
\text { index J }\end{array}$ & P-value \\
\hline \multicolumn{7}{|l|}{ One-dimensional model } \\
\hline miR-130b & $0.887(0.821-0.935)$ & 0.18 & 91.11 & 71.11 & 0.6222 & \\
\hline PDW,\% & $0.840(0.766-0.898)$ & 15.31 & 70.45 & 97.78 & 0.6823 & \\
\hline MLR & $0.814(0.738-0.876)$ & 0.15 & 80.90 & 75.56 & 0.5645 & \\
\hline $\mathrm{Hb}, \mathrm{g} / \mathrm{l}$ & $0.828(0.753-0.888)$ & 127.00 & 68.54 & 84.44 & 0.5298 & \\
\hline \multicolumn{7}{|l|}{ Dual-model } \\
\hline miR-130b-PDW & $0.922(0.863-0.961)$ & 0.74 & 82.02 & 88.89 & 0.7091 & $<0.0001^{\mathrm{a}}, 0.0005^{\mathrm{b}}$ \\
\hline miR-130b-MLR & $0.892(0.827-0.939)$ & 0.76 & 75.28 & 86.67 & 0.6195 & $<0.0001^{\mathrm{a}}, 0.1541^{\mathrm{c}}$ \\
\hline miR-130b-Hb & $0.937(0.882-0.972)$ & 0.56 & 89.89 & 86.67 & 0.7655 & $<0.0001^{\mathrm{a}}, 0.0014^{\mathrm{d}}$ \\
\hline PDW-MLR & $0.843(0.769-0.900)$ & 0.53 & 71.26 & 97.78 & 0.6904 & $0.5030^{b}, 0.5662^{c}$ \\
\hline PDW-Hb & $0.945(0.891-0.977)$ & 0.50 & 89.66 & 88.89 & 0.7854 & $0.0003^{\mathrm{b}}, 0.0001^{\mathrm{d}}$ \\
\hline MLR-Hb & $0.835(0.761-0.893)$ & 0.64 & 73.03 & 84.44 & 0.5748 & $0.6893^{\mathrm{c}}, 0.0940^{\mathrm{d}}$ \\
\hline \multicolumn{7}{|l|}{ Tri-model } \\
\hline miR-130b-PDW-MLR & $0.955(0.905-0.984)$ & 0.61 & 94.25 & 91.11 & 0.8536 & $0.0921^{\mathrm{e}}, 0.0091^{\mathrm{f}}, 0.0005^{\mathrm{g}}$ \\
\hline miR-130b-PDW-Hb & $0.976(0.933-0.995)$ & 0.77 & 90.80 & 97.78 & 0.8858 & $0.0602^{\mathrm{e}}, 0.0218^{\mathrm{h}}, 0.0341^{\mathrm{i}}$ \\
\hline miR-130b-MLR-Hb & $0.942(0.888-0.975)$ & 0.52 & 91.01 & 86.67 & 0.7768 & $0.0095^{\mathrm{f}}, 0.0697^{\mathrm{h}}, 0.0012^{\mathrm{j}}$ \\
\hline PDW-MLR-Hb & $0.947(0.894-0.978)$ & 0.55 & 89.66 & 91.11 & 0.8077 & $0.0004^{\mathrm{g}}, 0.2198^{\mathrm{i}}, 0.0002^{\mathrm{j}}$ \\
\hline \multicolumn{7}{|l|}{ Tetrad-model } \\
\hline miR-130b-PDW-MLR-Hb & $0.978(0.936-0.996)$ & 0.76 & 91.95 & 97.78 & 0.8973 & $0.0651^{\mathrm{k}}, 0.1465^{\mathrm{l}}, 0.0277^{\mathrm{m}}, 0.0316^{\mathrm{n}}$ \\
\hline
\end{tabular}

Table IV. Accuracy of miR-130b, PDW, MLR and Hb individually and combined for gastric cancer detection.

\begin{tabular}{|c|c|c|c|c|c|c|c|}
\hline Variable & Cut-off & Sensitivity, \% & Specificity, \% & Accuracy, $\%$ & $\mathrm{LR}^{+}$ & $\mathrm{LR}^{-}$ & DOR \\
\hline $\operatorname{miR}-130 b$ & 0.18 & 91.11 & 71.11 & 84.44 & 3.15 & 0.13 & 25.23 \\
\hline PDW, $\%$ & 15.31 & 70.45 & 97.78 & 72.39 & 3.43 & 0.39 & 8.71 \\
\hline MLR & 0.15 & 80.90 & 75.56 & 79.11 & 3.31 & 0.25 & 13.09 \\
\hline $\mathrm{Hb}, \mathrm{g} / \mathrm{l}$ & 127.00 & 68.54 & 84.44 & 73.88 & 4.40 & 0.37 & 11.82 \\
\hline miR-130b-PDW & 0.74 & 82.02 & 88.89 & 84.33 & 7.38 & 0.20 & 36.49 \\
\hline miR-130b-MLR & 0.764 & 75.28 & 86.67 & 79.11 & 5.65 & 0.29 & 19.80 \\
\hline miR-130b-Hb & 0.56 & 89.89 & 86.67 & 88.80 & 6.74 & 0.12 & 57.81 \\
\hline PDW-MLR & 0.53 & 71.26 & 97.78 & 80.17 & 32.10 & 0.29 & 109.21 \\
\hline PDW-Hb & 0.50 & 89.66 & 88.89 & 89.40 & 8.07 & 0.12 & 69.38 \\
\hline MLR-Hb & 0.64 & 73.03 & 84.44 & 76.89 & 4.69 & 0.32 & 14.69 \\
\hline miR-130b-PDW-MLR & 0.61 & 94.25 & 91.11 & 93.18 & 10.60 & 0.06 & 167.99 \\
\hline miR-130b-PDW-Hb & 0.77 & 90.80 & 97.78 & 93.18 & 40.90 & 0.09 & 434.71 \\
\hline miR-130b-MLR-Hb & 0.52 & 91.01 & 86.67 & 89.55 & 6.83 & 0.10 & 65.82 \\
\hline PDW-MLR-Hb & 0.55 & 89.66 & 91.11 & 90.15 & 10.09 & 0.11 & 88.87 \\
\hline miR-130b-PDW-MLR-Hb & 0.76 & 91.95 & 97.78 & 93.94 & 41.42 & 0.08 & 503.10 \\
\hline
\end{tabular}

$\mathrm{LR}^{+}$, positive likelihood ratio; LR', negative likelihood ratio; DOR, diagnostic odds ratio; miR-130b, microRNA-130b; PDW, platelet distribution width; MLR, monocyte-lymphocyte count ratio; Hb, hemoglobin. 
Table V. Crude odds ratio and adjusted odds ratio between circulating tumor marker levels and the risk of gastric cancer.

\begin{tabular}{lcccccccc}
\hline Indicators & $\beta$ & Crude odds ratio & HR (95\% CI) & Sig & $\beta$ & Adjusted odds ratio $^{\mathrm{a}}$ & HR (95\% CI) & Sig \\
\hline miR-130b & 0.900 & 0.406 & $0.301-0.548$ & 0.000 & 0.869 & 0.419 & $0.293-0.601$ & 0.000 \\
RDW-CV, $\%$ & 0.578 & 10.782 & $10.326-20.393$ & 0.000 & 0.574 & 1.776 & $1.197-6.744$ & 0.004 \\
MPV, fl & 0.519 & 10.680 & $10.173-20.405$ & 0.005 & 0.710 & 2.034 & $1.272-7.309$ & 0.003 \\
NLR & 0.802 & 20.229 & $10.460-30.404$ & 0.000 & 1.006 & 2.733 & $1.484-5.974$ & 0.001 \\
PLR & 0.008 & 10.008 & $10.003-10.013$ & 0.001 & 0.007 & 1.007 & $1.002-5.951$ & 0.008 \\
PDW,\% & -0.769 & 0.464 & $0.334-0.643$ & 0.000 & -0.702 & 0.496 & $0.353-0.696$ & 0.000 \\
N\% & 0.054 & 10.055 & $10.022-10.090$ & 0.001 & 0.057 & 1.059 & $1.014-6.421$ & 0.010 \\
M\% & 0.415 & 10.514 & $10.194-10.918$ & 0.001 & 0.412 & 1.511 & $1.126-2.027$ & 0.006 \\
N\#,109/1 & 0.026 & 10.027 & $0.930-10.133$ & 0.601 & 0.051 & 1.052 & $0.963-1.150$ & 0.262 \\
M\#,10/1 & 0.494 & 10.639 & $0.398-60.753$ & 0.494 & 0.499 & 1.646 & $0.342-7.926$ & 0.534 \\
Hb, g/l & -0.063 & 0.939 & $0.914-0.964$ & 0.000 & -0.070 & 0.933 & $0.903-0.964$ & 0.000 \\
Hct, $\%$ & -0.191 & 0.826 & $0.757-0.902$ & 0.000 & -0.201 & 0.818 & $0.737-0.907$ & 0.000 \\
MLR & -0.219 & 1.245 & $0.607-2.554$ & 0.550 & -0.188 & 0.829 & $0.477-1.439$ & 0.505 \\
L\% & -0.103 & 0.902 & $0.802-0.943$ & 0.000 & -0.100 & 0.905 & $0.858-0.954$ & 0.000 \\
L\#,10/1 & -1.041 & 0.353 & $0.198-0.630$ & 0.000 & -0.923 & 0.397 & $0.203-0.776$ & 0.007 \\
\hline
\end{tabular}

${ }^{a}$ Adjustment for age and sex. HR, hazard ratio; 95\% CI, 95\% confidence interval; miR-130b, microRNA-130b; RDW-CV, red blood cell distribution width-coefficient of variation; MPV, mean platelet volume; NLR, neutrophil to lymphocyte ratio; PLR, platelet to lymphocyte ratio; PDW, platelet distribution width; N\%, neutrophil percentage; M\%; N\#, neutrophil count; M\#, monocyte count; Hb, hemoglobin; Hct, hematocrit; MLR, monocyte to lymphocyte ratio; L\%, lymphocyte percentage; L\#, lymphocyte count.

stage and MPV, L\#, L\%, Hb and Hct were negatively correlated with cancer stage. However, PDW, M\%, M\#, MLR and miR-130b were not correlated with cancer stage. In addition, the levels of circulating RDW-CV, PLR, NLR, and N\% were significantly higher in advanced stages (III and IV) than the earlier pathologic stages (I and II) of GC. By contrast, the levels of circulating $\mathrm{L} \%, \mathrm{Hb}$, Hct and L\# were significantly lower in advanced stages than in the earlier pathological stages of GC. However, there was no difference in miR-130b between the two groups (Fig. 5). Since the levels of RDW-CV, PLR, NLR and N\% were positively correlated with tumor pathological characteristics (TNM stage), it is reasonable to hypothesize that these biomarkers may be associated with GC metastasis.

Integrative diagnosis model for discriminating EGC. To evaluate the role of circulating biomarkers in the diagnosis of early GC (TNM I-II), the present study further analyzed the levels of these markers (Fig. 6; Table VI). As shown in Fig. 6A-C, G-I and M-O, the circulating levels of mi-130b, NLR, RDW-CV, PDW, M\%, R\#, Hct, Hb and MLR differed significantly between the early stage group and the NC group. The results in Fig. 6D-F, J-L and P-R demonstrate the diagnostic efficiency of circulating miR-130b, NLR, RDW-CV, PDW, M\%, R\#, Hct, Hb and MLR individually for discriminating patients with EGC from controls. As one-dimensional models, miRNA-130b, RDW-CV, NLR, PDW, M\%, R\#, Hb, Hct, and MLR individually yielded AUC values of 0.9110 , $0.7130,0.6491,0.8037,0.7367,0.7019,0.7745,0.6935$ and 0.7610 , respectively. These results indicated that miR-130b, NLR, RDW-CV, PDW, M\%, R\#, Hct, Hb and MLR could be used as novel biomarkers for the early diagnosis of GC.
To further explore the diagnostic efficacy of joint markers, the biomarkers with an AUC $>0.77$ (miR-130b, PDW and $\mathrm{Hb}$ ) were further considered for combinations. Among the two-dimensional models (i.e., the models with two biomarkers), miR-130b-PDW yielded the greatest AUC (0.956), followed by miR-130b-Hb (0.923) and PDW-Hb (0.906). The combination of miR-130b with $\mathrm{Hb}$ had a significantly larger AUC value (0.923; 0.833-0.974) compared with $\mathrm{Hb}$ alone $(\mathrm{P}=0.0181)$, and the combination of PDW and $\mathrm{Hb}$ had a significantly larger AUC value (0.906; 0.812-0.963) compared with $\mathrm{Hb}$ alone $(\mathrm{P}=0.0464)$. The tri-model (miR-130b-PDW-Hb) had an AUC of 0.960 (0.883-0.993). However, there were no significant differences in the AUC values between the tri-model and dual-models $(\mathrm{P}=0.2250, \mathrm{P}=0.4331$ and $\mathrm{P}=0.0940)$.

Diagnostic performance of tri-dimensional biomarkers for patients with EGC. The corresponding diagnostic accuracy parameters, including sensitivity, specificity, accuracy, $\mathrm{LR}^{+}$, LR $^{-}$and DOR, are shown in Table VII. miR-130b and $\mathrm{Hb}$ had high sensitivities (95.56 and 83.33\%, respectively), RDW-CV, PDW, R\# and Hct had high specificities (80.00, 97.78, 97.78 and $93.33 \%$, respectively), and miR-130b, PDW and R\# had high accuracy $(82.15,91.31$ and $88.27 \%$, respectively) for distinguishing early stage cancer from NCs. The specificity and accuracy increased when miR-130b and $\mathrm{Hb}$ were combined, and the sensitivity and accuracy increased when miR-130b and PDW were combined. The combination of miR-130b-PDW-Hb could not improve the sensitivity, specificity and accuracy for the diagnosis of EGC in comparison with the two-dimensional model of miR-130b-PDW. Furthermore, the combination of miR-130b and PDW yielded the largest sensitivity, specificity, accuracy, $\mathrm{LR}^{+}$and DOR values, and the lowest $\mathrm{LR}^{-}$value. 
A

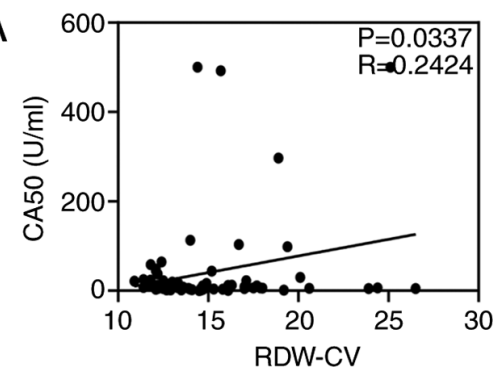

D

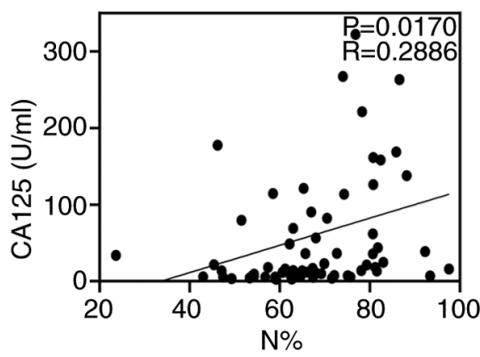

G

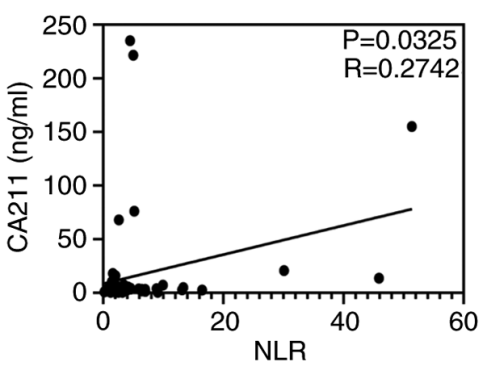

J

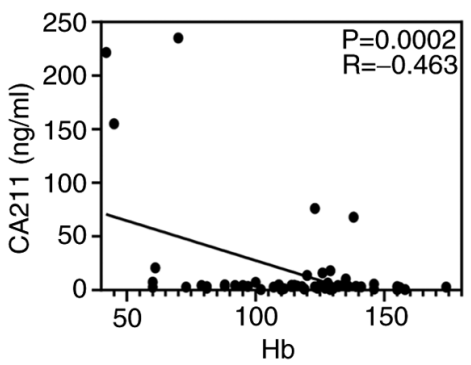

M

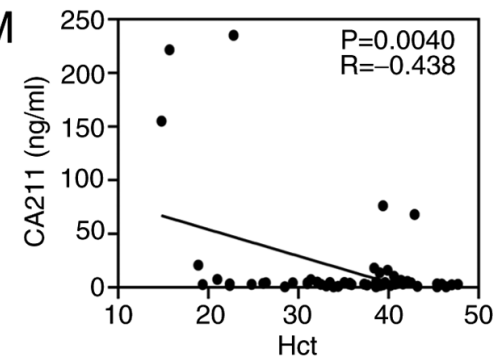

B

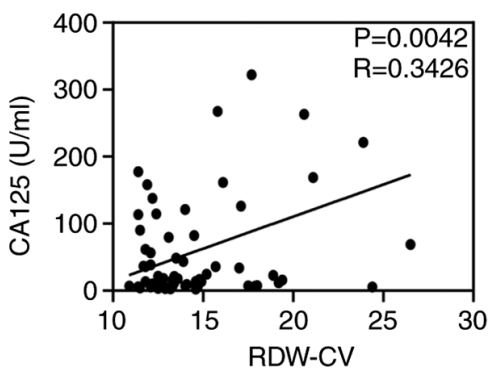

E

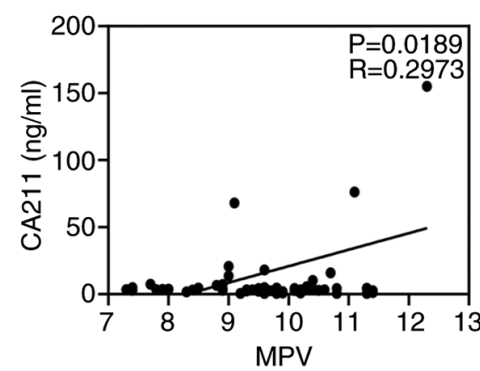

$\mathrm{H}$

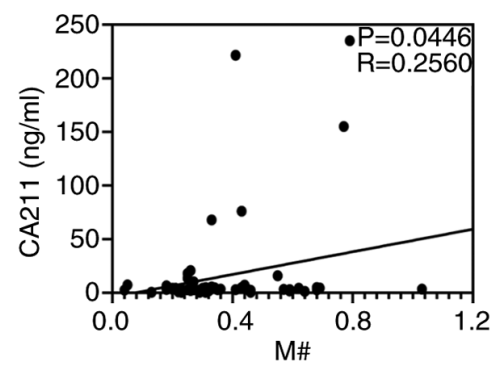

K

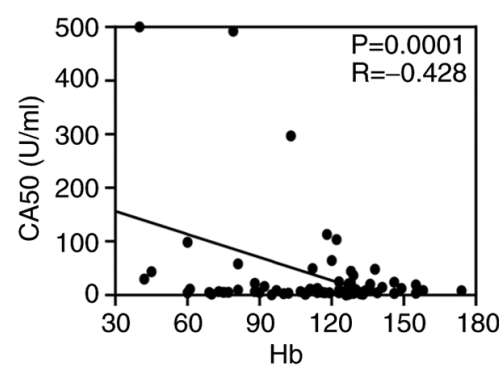

$\mathrm{N}$

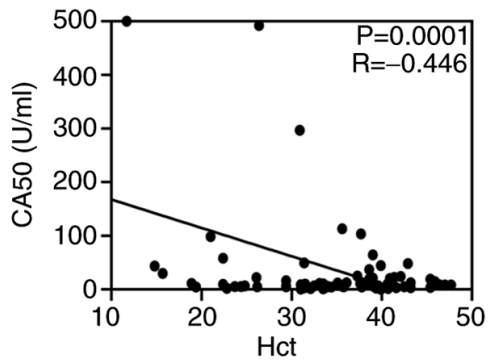

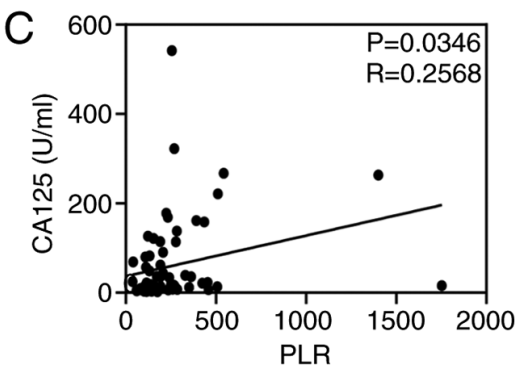

F
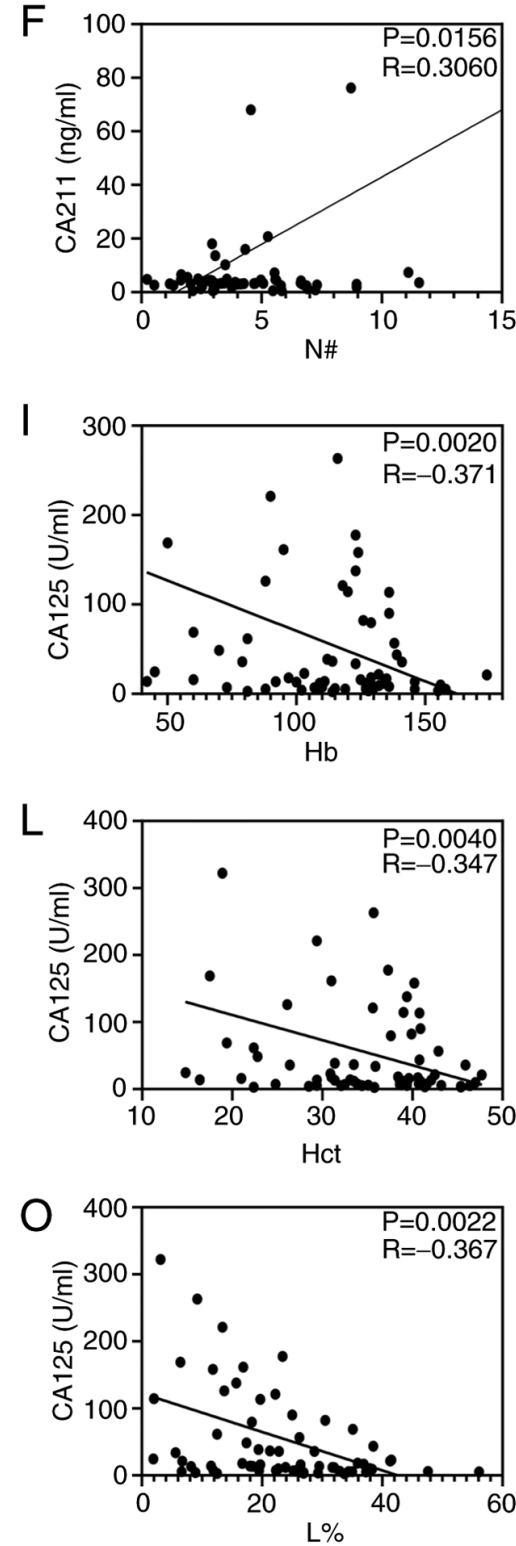

Figure 3. Circulating biomarkers and conventional gastric cancer markers analyzed using Pearson's correlation. Correlation analysis of RDW-CV with (A) CA50 and (B) CA125. Correlation analysis of CA125 with (C) PLR and (D) N\%. Correlation between (E) MPV, (F) N\#, (G) NLR and (H) M\# and CA211. Continuous data for $\mathrm{Hb}$ with (I) CA125, (J) CA211 and (K) CA50. Continuous data for Hct with (L) CA125, (M) CA211 and (N) CA50. (O) Correlation analysis of L\% with CA125. Hb, hemoglobin; Hct, hematocrit; L\%, lymphocyte percentage; MPV, mean platelet volume; M\#, monocyte count; N\#, neutrophil count; NLR, neutrophil to lymphocyte ratio; PLR, platelet to lymphocyte ratio; RDW-CV, red blood cell distribution width-coefficient of variation.

These results indicated that miR-130b-PDW had great diagnostic value for EGC and could be used as a non-invasive diagnostic model for EGC.

Integrative diagnosis model for discriminating precancerous gastric lesions. Based on the differences in RDW-CV,
MPV/PC ratio, MLR, NLR, PDW, L\%, M\%, M\# and $\mathrm{Hb}$ between the Pre and NC groups (Table I), the diagnostic analyses of single or combinations of selected differentially expressed biomarkers were conducted (Table VIII; Fig. 7). As shown in Table VIII, the AUC values for miR-130b, RDW-CV, MPV/PC ratio, MLR, NLR, PDW, L \%, M\%, M\# 
A
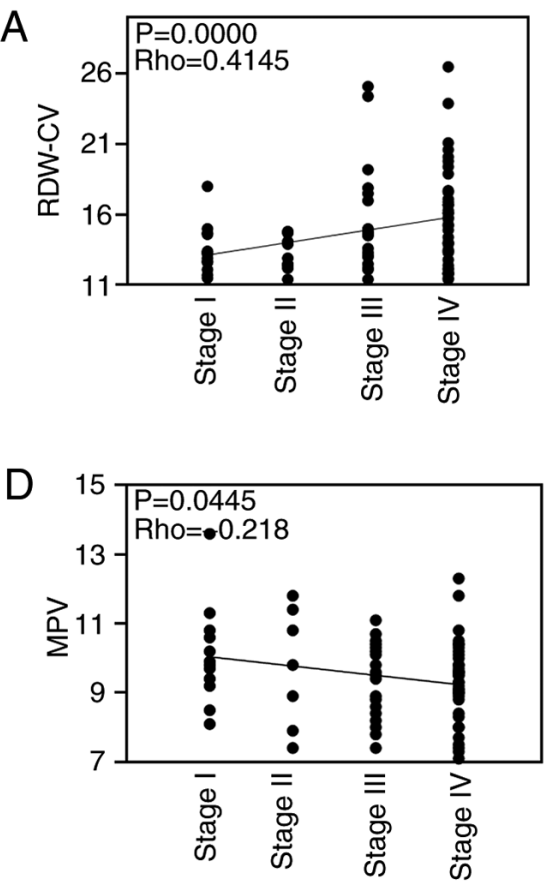

G
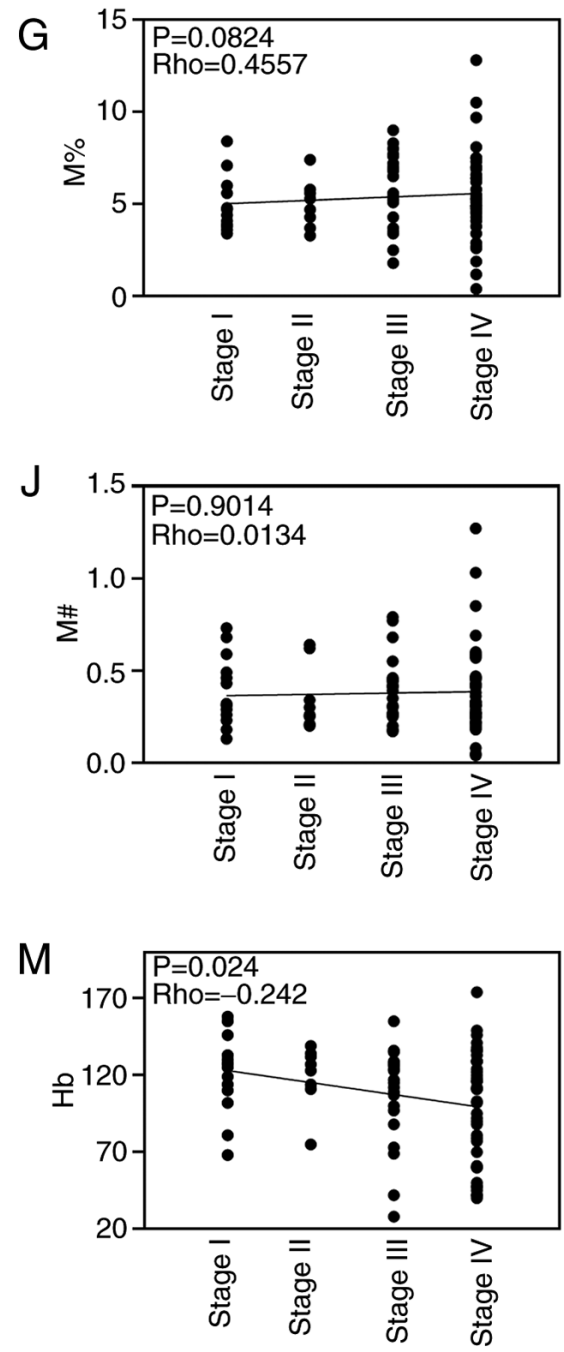

B

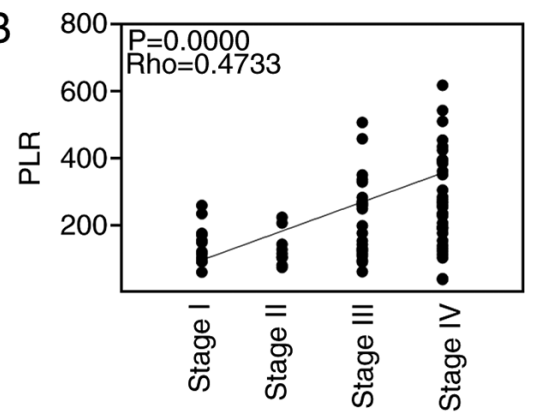

E

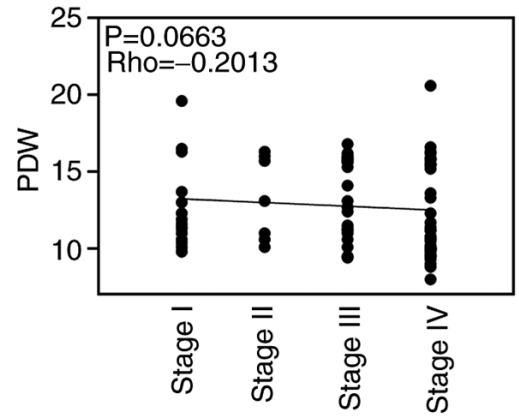

$\mathrm{H}$

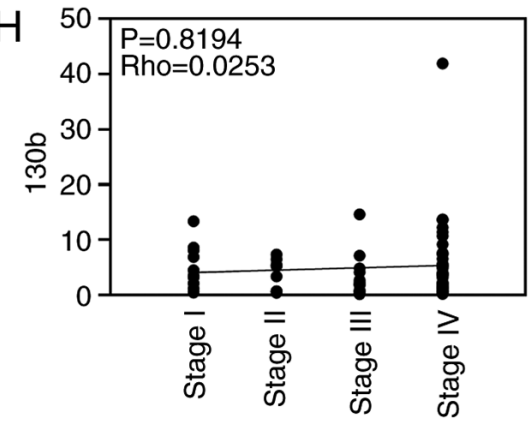

K

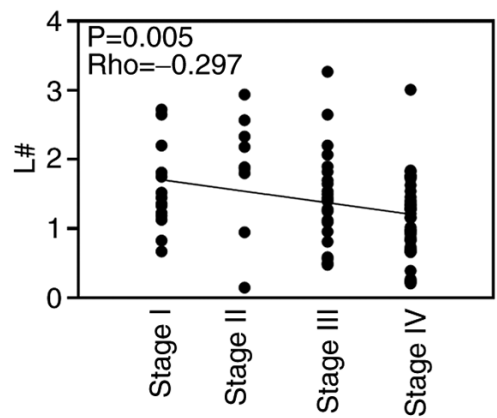

N

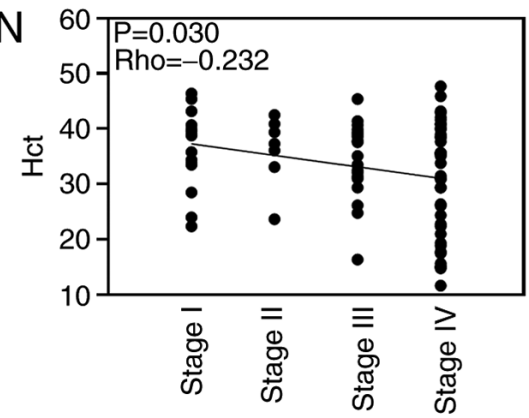

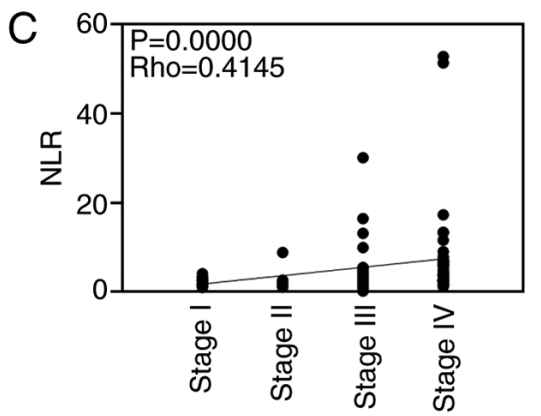

$F$
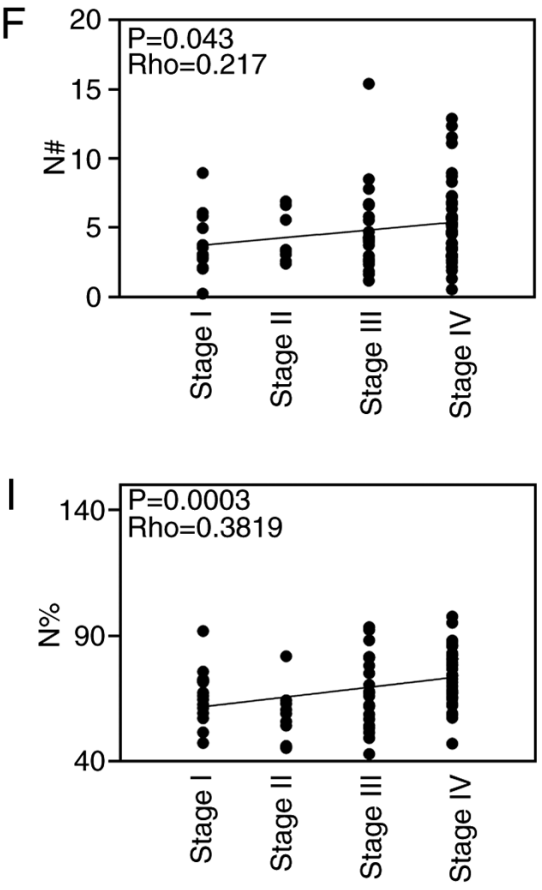

L
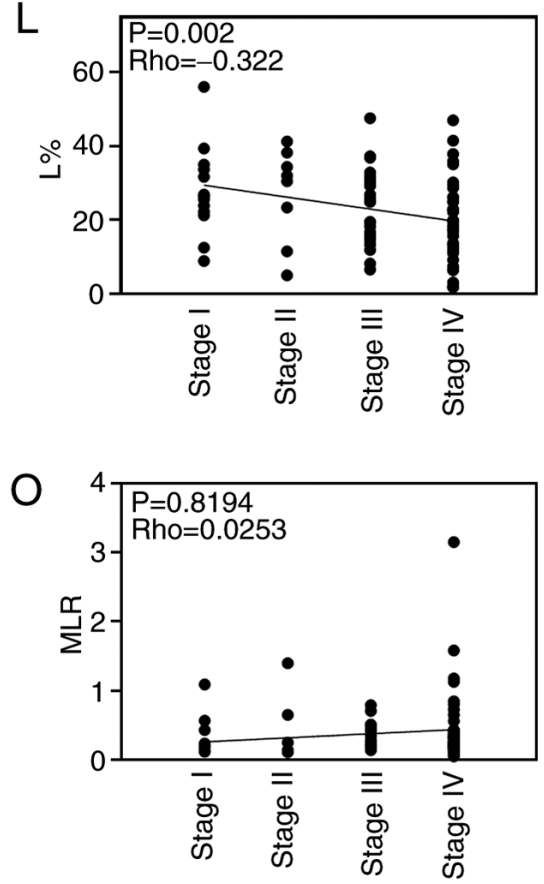

Figure 4. Spearman's correlation analysis of blood biochemical indexes and cancer stage. Analysis of correlation between blood biochemical indexes, including (A) RDW-CV, (B) PLR, (C) NLR, (D) MPV, (E) PDW, (F) N\#, (G) M\%, (H) miR-130b, (I) N\%, (J) M\#, (K) L\#, (L) L\%, (M) Hb, (N) Hct and (O) MLR, and cancer stage. Hb, hemoglobin; Hct, hematocrit; L\%, lymphocyte percentage; L\#, lymphocyte count; miR-130b, microRNA-130b; MLR, monocyte to lymphocyte ratio; MPV, mean platelet volume; M\%, monocyte percentage; M\#, monocyte count; N\%, neutrophil percentage; N\#, neutrophil count; NLR, neutrophil to lymphocyte ratio; PDW, platelet distribution width; PLR, platelet to lymphocyte ratio; RDW-CV, red blood cell distribution width-coefficient of variation. 
A

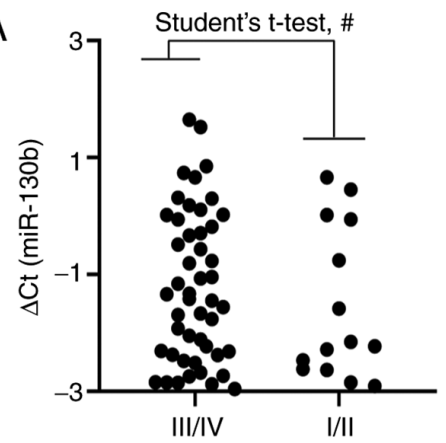

D
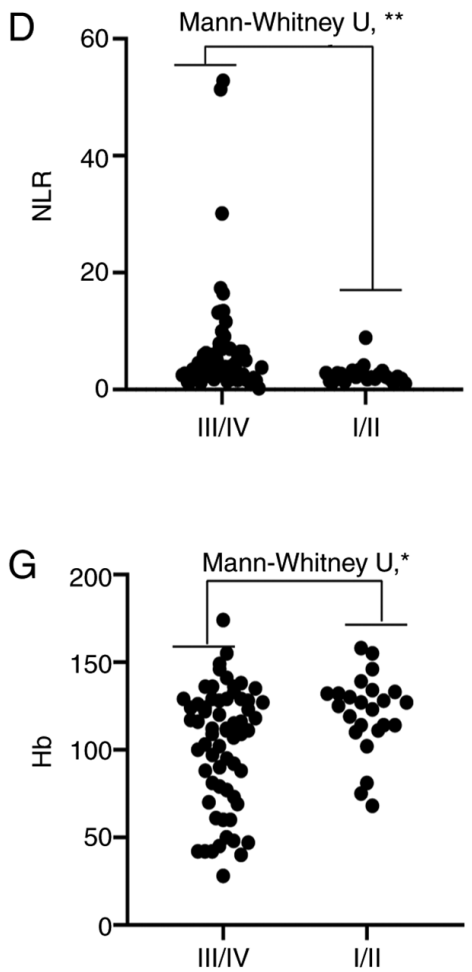

B
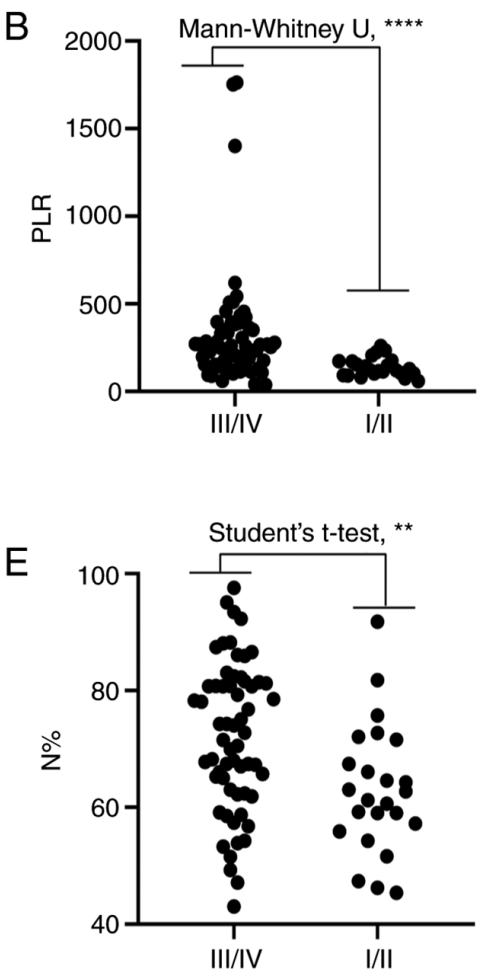

$\mathrm{H}$

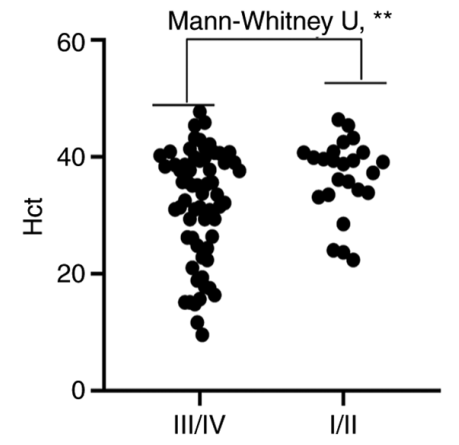

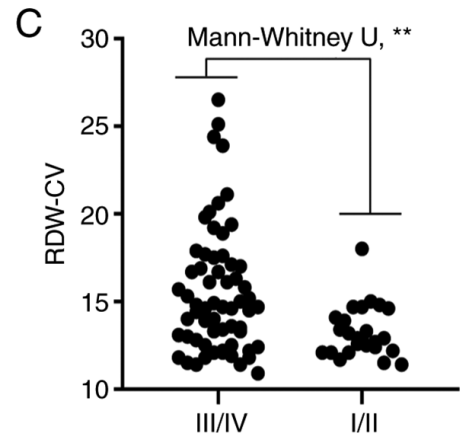

F

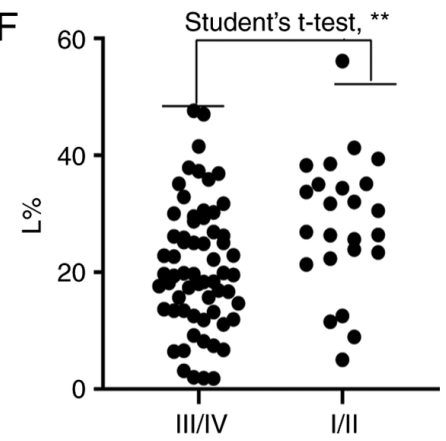

I

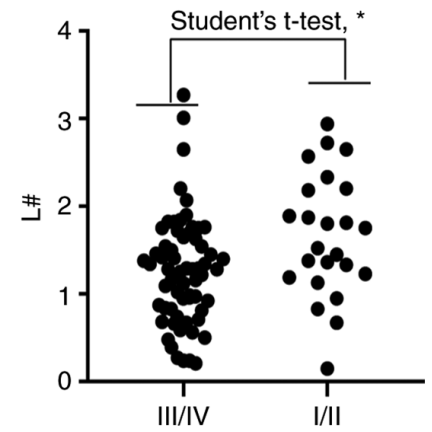

Figure 5. Comparison of circulating biomarker expression between the early stages (I/II) and advanced pathological stages (III/IV) of gastric cancer. (A) $\Delta \mathrm{Cq}$ value of miR-130b, (B) PLR, (C) RDW-CV, (D) NLR, (E) N\%, (F) L\%, (G) Hb, (H) Hct and (I) L\#. Unpaired t-test or Mann-Whitney U test was used to determine statistical significance at $\left({ }^{*} \mathrm{P}>0.05,{ }^{*} \mathrm{P}<0.05,{ }^{* *} \mathrm{P}<0.01\right.$ and $\left.{ }^{* * * * *} \mathrm{P}<0.0001\right)$. Hb, hemoglobin; Hct, hematocrit; L\%, lymphocyte percentage; L\#, lymphocyte count; miR-130b, microRNA-130b; N\%, neutrophil percentage; NLR, neutrophil to lymphocyte ratio; PLR, platelet to lymphocyte ratio; RDW-CV, red blood cell distribution width-coefficient of variation.

and $\mathrm{Hb}$ were $0.700,0.649,0.687,0.713,0.616,0.811,0.638$, $0.649,0.657$ and 0.679 , respectively. To further explore the diagnostic efficacy of joint models, the biomarkers with an AUC $>0.70$ (miR-130b, PDW and MLR) were further considered for combinations. Among the two-dimensional models (i.e., the models with two biomarkers), miR-130b-PDW yielded the greatest AUC (0.896), followed by MLR-PDW (0.816) and miR-130b-MLR (0.687). The dual-model miR-130b-PDW had a significantly larger AUC 0.896 (0.827-0.944) compared with miR-130b and PDW, respectively. The tri-model (miR-130b-PDW-MLR) yielded an AUC value of 0.896 (0.827-0.944), which was larger than those of the dual-models of miR-130b-MLR and MLR-PDW. However, there were no significant differences in the AUC values between the tri-model (miR-130b-MLR-PDW) and the dual-model (miR-130b-PDW).
Diagnostic performance of tri-dimensional biomarkers for patients with Pres. The corresponding diagnostic accuracy parameters, including sensitivity, specificity, accuracy, $\mathrm{LR}^{+}$, LR $^{-}$and DOR, are shown in Table IX. MLR and PDW had high sensitivities for distinguishing Pres from NCs (77.92 and $72.37 \%$, respectively), miR-130b, NLR and L\% had high specificities (93.30, 100.00 and $100.00 \%$, respectively), and PDW had high accuracy (81.74\%). The sensitivity and accuracy increased when miR-130b and PDW were combined. Furthermore, the combination of MLR-PDW yielded the largest $\mathrm{LR}^{+}$and DOR values. However, the combination of miR-130b-MLR-PDW could not improve the sensitivity, specificity and accuracy for the diagnosis of precancerous gastric lesions. These results indicated that the dual-model of miR-130b-PDW was an appropriate and non-invasive diagnostic model for Pres. 

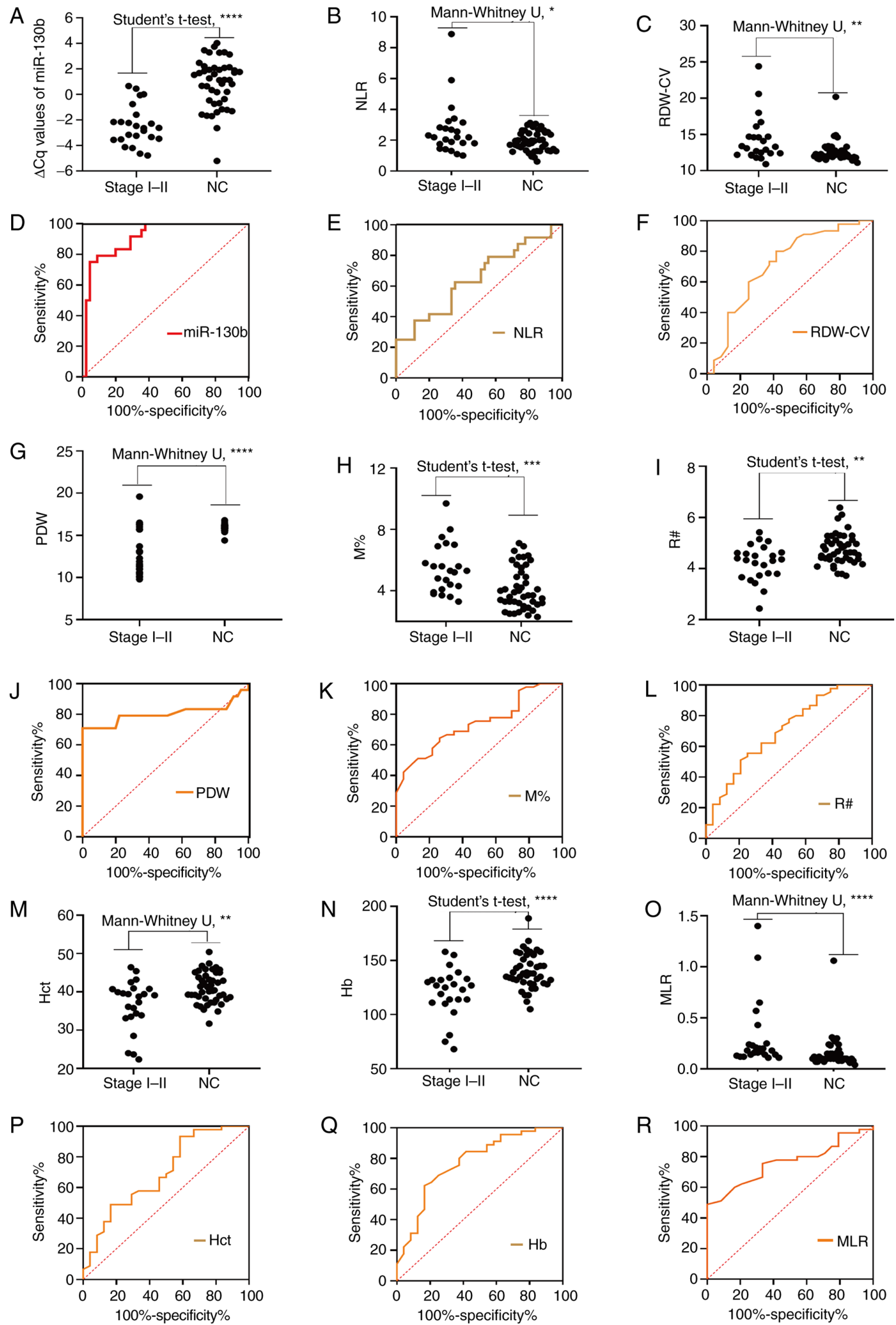

Figure 6. Diagnosis of early stages (I/II) of gastric cancer. Comparison of circulating biomarker expression between the early stages (I/II) of gastric cancer and NCs. (A) $\triangle$ Cq value of miR-130b, (B) NLR, (C) RDW-CV, (G) PDW, (H) M\%, (I) R\#, (M) Hct, (N) Hb and (O) MLR. Unpaired t-test or Mann-Whitney $\mathrm{U}$ test was used to determine statistical significance $\left({ }^{*} \mathrm{P}<0.05,{ }^{* *} \mathrm{P}<0.01,{ }^{* * *} \mathrm{P}<0.001\right.$ and $\left.{ }^{* * * *} \mathrm{P}<0.0001\right)$. Area under the curve of the expression of circulating biomarkers for early gastric cancer diagnosis. (D) $\triangle$ Cq value of miR-130b, (E) NLR, (F) RDW-CV, (J) PDW, (K) M\%, (L) R\#, (P) Hct, (Q) Hb and (R) MLR. Hb, hemoglobin; Hct, hematocrit; miR-130b, microRNA-130b; MLR, monocyte to lymphocyte ratio; M\%, monocyte percentage; NC, healthy control; NLR, neutrophil to lymphocyte ratio; PDW, platelet distribution width; R\#, red blood cell count; RDW-CV, red blood cell distribution width-coefficient of variation. 
Table VI. Receiver operating characteristic analysis of circulating biomarkers individually and combined for early stage gastric cancer detection.

\begin{tabular}{|c|c|c|}
\hline Models & AUC $(95 \% \mathrm{CI})$ & P-value \\
\hline \multicolumn{3}{|l|}{ One-dimensional model } \\
\hline miR-130b & $0.9110(0.8417-0.9805)$ & \\
\hline RDW-CV,\% & $0.7130(0.5770-0.8490)$ & \\
\hline NLR & $0.6491(0.5082-0.7900)$ & \\
\hline PDW,\% & $0.8037(0.6616-0.9458)$ & \\
\hline $\mathrm{M} \%$ & 0.7367 (0.6196-0.8539) & \\
\hline $\mathrm{R} \#, 10^{12} / 1$ & $0.7019(0.5715-0.8322)$ & \\
\hline $\mathrm{Hb}, \mathrm{g} / \mathrm{l}$ & $0.7745(0.6563-0.8928)$ & \\
\hline Hct, $\%$ & $0.6935(0.5601-0.8269)$ & \\
\hline MLR & $0.7610(0.6430-0.8560)$ & \\
\hline \multicolumn{3}{|l|}{ Dual-model } \\
\hline miR-130b-PDW & $0.9560(0.877-0.991)$ & $0.3775^{\mathrm{a}} ; 0.0576^{\mathrm{b}}$ \\
\hline miR-130b-Hb & $0.9230(0.833-0.974)$ & $0.7412^{\mathrm{a}} ; 0.0181^{\mathrm{c}}$ \\
\hline PDW-Hb & $0.9060(0.812-0.963)$ & $0.1731^{\mathrm{b}} ; 0.046^{4 \mathrm{c}}$ \\
\hline \multicolumn{3}{|l|}{ Tri-model } \\
\hline miR-130b-PDW-Hb & $0.960 \quad(0.883-0.993)$ & $0.2250^{\mathrm{d}} ; 0.4331^{\mathrm{e}} ; 0.0940^{\mathrm{f}}$ \\
\hline
\end{tabular}

Table VII. Accuracy of miR-130b, PDW and Hb individually and combined for early stage gastric cancer detection.

\begin{tabular}{|c|c|c|c|c|c|c|c|c|}
\hline Variable & Cut-off & Youden index $\mathbf{J}$ & Sensitivity, $\%$ & Specificity, \% & Accuracy, $\%$ & $\mathrm{LR}^{+}$ & $\mathrm{LR}^{-}$ & DOR \\
\hline miR-130b & -2.15 & 0.71 & 95.56 & 75.00 & 82.15 & 3.82 & 0.06 & 64.57 \\
\hline RDW-CV,\% & 12.80 & 0.38 & 58.33 & 80.00 & 72.46 & 2.92 & 0.52 & 5.60 \\
\hline NLR & 2.04 & 0.27 & 62.50 & 64.44 & 63.77 & 1.76 & 0.58 & 3.02 \\
\hline PDW, $\%$ & 15.31 & 0.77 & 79.17 & 97.78 & 91.31 & 35.66 & 0.21 & 167.41 \\
\hline M\% & 4.20 & 0.38 & 73.91 & 64.44 & 67.64 & 2.08 & 0.40 & 5.13 \\
\hline $\mathrm{R} \#, 10^{12} / 1$ & 4.61 & 0.31 & 70.45 & 97.78 & 88.27 & 31.73 & 0.30 & 105.01 \\
\hline $\mathrm{Hb}, \mathrm{g} / \mathrm{l}$ & 134 & 0.46 & 83.33 & 62.22 & 69.56 & 2.21 & 0.27 & 8.23 \\
\hline Hct, $\%$ & 36.10 & 0.35 & 41.67 & 93.33 & 75.36 & 6.25 & 0.62 & 10.00 \\
\hline MLR & 0.10 & 0.49 & 100.00 & 48.89 & 66.67 & 1.95 & - & - \\
\hline miR-130b-PDW & 0.26 & 0.94 & 95.83 & 97.78 & 97.10 & 43.17 & 0.04 & $1,012.19$ \\
\hline miR-130b-Hb & 0.40 & 0.74 & 83.33 & 91.11 & 88.40 & 9.37 & 0.18 & 51.23 \\
\hline PDW-Hb & 0.31 & 0.79 & 83.33 & 95.56 & 91.31 & 18.77 & 0.17 & 107.59 \\
\hline miR-130b-PDW-Hb & 0.29 & 0.94 & 95.83 & 97.78 & 97.10 & 43.17 & 0.04 & $1,012.19$ \\
\hline
\end{tabular}

$\mathrm{LR}^{+}$, positive likelihood ratio; $\mathrm{LR}^{+}$, negative likelihood ratio; DOR, diagnostic odds ratio; miR-130b, microRNA-130b; RDW-CV, red blood cell distribution width-coefficient of variation; NLR, neutrophil to lymphocyte ratio; PDW, platelet distribution width; M\%, monocyte percentage; R\#, red blood cell count; Hb, hemoglobin; Hct, hematocrit; MLR, monocyte to lymphocyte ratio.

Baseline characteristics. A total of 90 patients with $\mathrm{GC}$ were included in the present study, including 46 men and 44 women, with a mean age of 65 years (range, 36-89 years). The age range was 29-88 (mean, 64.5 years) years for the 90 patients with Pres, including 48 male patients and 42 female patients. A total of 45 NCs (age range, 39-80 years) with a mean age of 65 years, including 21 male patients and 24 female patients, were included. In 
Table VIII. Receiver operating characteristic analysis of circulating biomarkers individually and combined for precancerous lesion detection.

\begin{tabular}{|c|c|c|}
\hline Models & AUC (95\% CI) & P-value \\
\hline \multicolumn{3}{|l|}{ One-dimensional model } \\
\hline miR-130b & $0.700(0.613-0.788)$ & \\
\hline RDW-CV, \% & $0.649(0.557-0.733)$ & \\
\hline MPV/PC ratio & $0.687(0.597-0.768)$ & \\
\hline MLR & $0.713(0.624-0.791)$ & \\
\hline NLR & $0.616(0.524-0.702)$ & \\
\hline PDW, \% & $0.811(0.730-0.877)$ & \\
\hline $\mathrm{L} \%$ & $0.638(0.546-0.723)$ & \\
\hline $\mathrm{M} \%$ & $0.649(0.558-0.733)$ & \\
\hline $\mathrm{M \#} 10^{9} / 1$ & $0.657(0.565-0.741)$ & \\
\hline $\mathrm{Hb}, \mathrm{g} / \mathrm{l}$ & $0.679(0.588-0.761)$ & \\
\hline \multicolumn{3}{|l|}{ Dual-model } \\
\hline miR-130b-MLR & $0.687(0.597-0.768)$ & $0.8154^{\mathrm{a}} ; 0.7273^{\mathrm{b}}$ \\
\hline miR-130b-PDW & $0.896(0.827-0.944)$ & $<0.0001^{\mathrm{a}} ; 0.0028^{\mathrm{c}}$ \\
\hline MLR-PDW & $0.816(0.736-0.881)$ & $0.1069^{\mathrm{b}} ; 0.0832^{\mathrm{c}}$ \\
\hline \multicolumn{3}{|l|}{ Tri-model } \\
\hline miR-130b-MLR-PDW & $0.896(0.827-0.944)$ & $0.0001^{\mathrm{d}} ; 0.6849^{\mathrm{e}} ; 0.0049^{\mathrm{f}}$ \\
\hline
\end{tabular}

Table IX. Accuracy of circulating biomarkers individually and combined for Pre detection.

\begin{tabular}{|c|c|c|c|c|c|c|c|c|c|}
\hline Pre vs. NC & AUC $(95 \%$ CI) & Cut-off & $\begin{array}{l}\text { Youden } \\
\text { index } \mathrm{J}\end{array}$ & $\begin{array}{c}\text { Sensitivity, } \\
\%\end{array}$ & $\begin{array}{c}\text { Specificity, } \\
\%\end{array}$ & $\begin{array}{c}\text { Accuracy, } \\
\%\end{array}$ & $\mathrm{LR}^{+}$ & $\mathrm{LR}^{-}$ & DOR \\
\hline RDW-CV & $0.649(0.557-0.733)$ & 12.40 & 0.2808 & 63.64 & 64.44 & 63.94 & 1.79 & 0.56 & 3.17 \\
\hline $\mathrm{MPV} / \mathrm{PC}$ ratio & $0.687(0.597-0.768)$ & 0.04 & 0.2973 & 51.95 & 77.78 & 61.48 & 2.34 & 0.62 & 3.78 \\
\hline MLR & $0.713(0.624-0.791)$ & 0.12 & 0.3792 & 77.92 & 60.00 & 71.31 & 1.95 & 0.37 & 5.29 \\
\hline NLR & $0.616(0.524-0.702)$ & 3.12 & 0.3506 & 35.06 & 100.00 & 59.01 & - & 0.65 & - \\
\hline PDW & $0.811(0.730-0.877)$ & 15.31 & 0.7015 & 72.37 & 97.78 & 81.74 & 32.60 & 0.28 & 115.37 \\
\hline $\mathrm{L} \%$ & $0.638(0.546-0.723)$ & 21.71 & 0.3377 & 33.77 & 100.00 & 58.20 & - & 0.66 & - \\
\hline M\% & $0.649(0.558-0.733)$ & 4.50 & 0.2547 & 57.14 & 68.89 & 61.47 & 1.84 & 0.62 & 2.95 \\
\hline M\# & $0.657(0.565-0.741)$ & 0.25 & 0.2974 & 69.74 & 60.00 & 66.15 & 1.74 & 0.50 & 3.46 \\
\hline $\mathrm{Hb}$ & $0.679(0.588-0.761)$ & 127.00 & 0.3250 & 48.05 & 84.44 & 61.47 & 3.09 & 0.62 & 5.02 \\
\hline miR-130b-MLR & $0.687(0.597-0.768)$ & 0.68 & 0.4343 & 54.55 & 88.89 & 67.22 & 4.91 & 0.51 & 9.60 \\
\hline miR-130b-PDW & $0.896(0.827-0.944)$ & 0.51 & 0.7623 & 82.89 & 93.33 & 86.77 & 12.43 & 0.18 & 67.79 \\
\hline MLR-PDW & $0.816(0.736-0.881)$ & 0.51 & 0.7146 & 73.68 & 97.78 & 82.64 & 33.19 & 0.27 & 123.30 \\
\hline miR-130b-MLR-PDW & $0.896(0.827-0.944)$ & 0.51 & 0.7623 & 82.89 & 93.33 & 86.77 & 12.43 & 0.18 & 67.79 \\
\hline
\end{tabular}

Pre, precancerous lesions; NC, healthy control; AUC, area under the curve; 95\% CI, 95\% confidence interval; $\mathrm{LR}^{+}$, positive likelihood ratio; $\mathrm{LR}^{-}$, negative likelihood ratio; DOR, diagnostic odds ratio; miR-130b, microRNA-130b; RDW-CV, red blood cell distribution width-coefficient of variation; MPV/PC, mean platelet volume to platelet count ratio; MLR, monocyte to lymphocyte count ratio; NLR, neutrophil to lymphocyte ratio; PDW, platelet distribution width; L\%, lymphocyte percentage; M\%, monocyte percentage; M\#, monocyte count; Hb, hemoglobin.

the present study, no significant differences were observed in age and sex $(\mathrm{P}=0.9598$ and $\mathrm{P}=0.7658$, respectively). The clinical characteristics of all patients are summarized in Table X. 

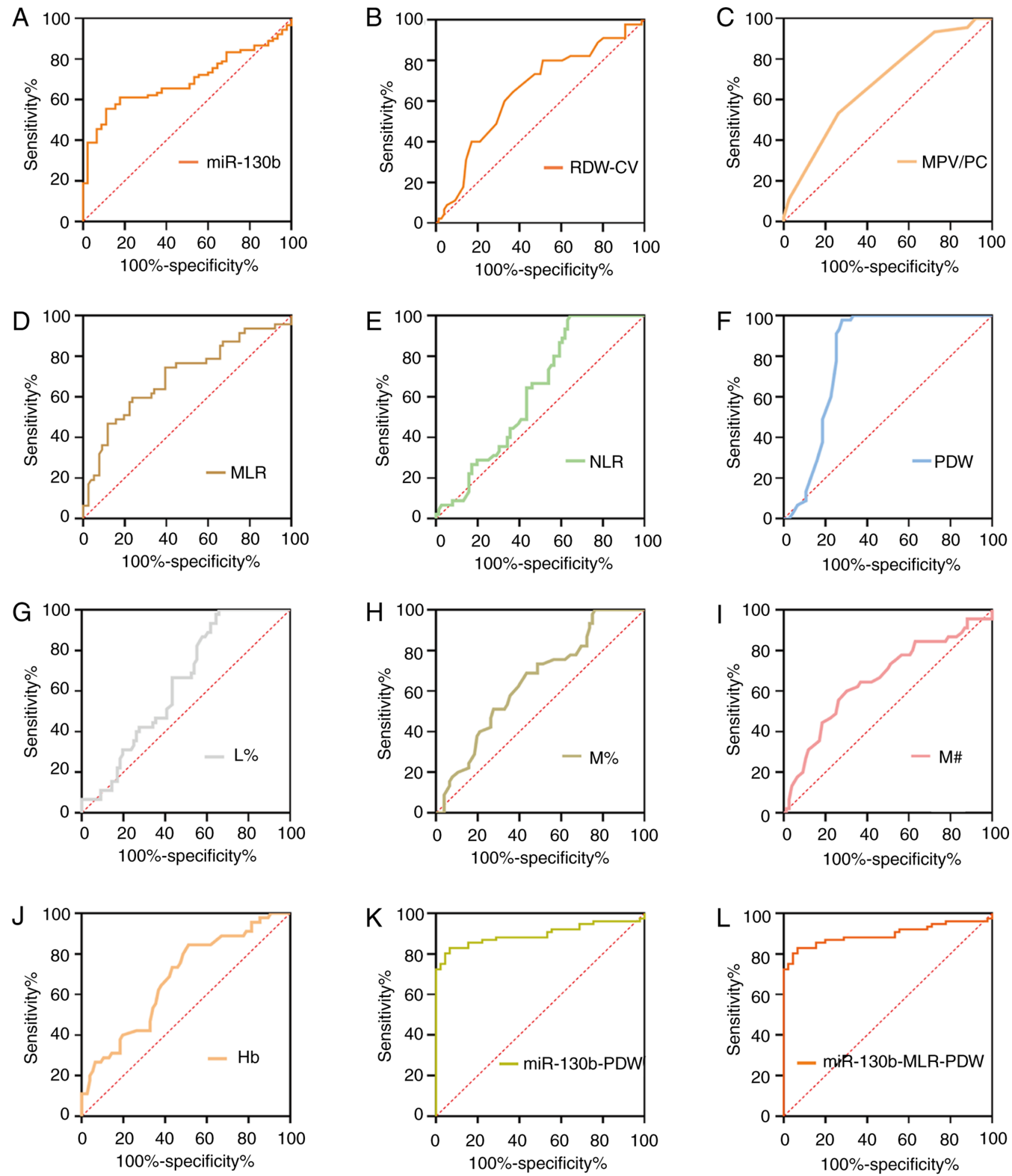

Figure 7. ROC curve analyses of circulating biomarkers for Pres. ROC analysis for detection of Pres using (A) miR-130b, (B) RDW-CV, (C) MPV/PC, (D) MLR, (E) NLR, (F) PDW, (G) L\%, (H) M\%, (I) M\#, (J) Hb, (K) miR-130b-PDW and (L) miR-130b-MLR-PDW. Hb, hemoglobin; L\%, lymphocyte percentage; miR-130b, microRNA-130b; MLR, monocyte to lymphocyte ratio; MPV/PC, mean platelet volume to platelet count ratio; M\%, monocyte percentage; M\#, monocyte count; NLR, neutrophil to lymphocyte ratio; PDW, platelet distribution width; RDW-CV, red blood cell distribution width-coefficient of variation; ROC, receiver operating characteristic; Pres, precancerous gastric lesions.

\section{Discussion}

The early diagnosis of GC is of great significance. In the present study, an integrated analysis was performed and it was revealed that plasma miR-130b and partial parameters of blood routine, such as PDW, RDW, MLR, NLR, and PLR, were dysregulated in Pres, EGC and GC. Furthermore, the early diagnostic value of miR-130b and blood routine parameters alone or in combination was explored and an optimal diagnostic model for GC, EGC and Pre was identified. 
Table X. Clinicopathological characteristics of all individuals by subgroup.

\begin{tabular}{|c|c|c|c|c|}
\hline Characteristics & $\mathrm{GC}(\mathrm{n}=90)$ & Pre $(n=90)$ & $\mathrm{NC}(\mathrm{n}=45)$ & P-value \\
\hline Mean age $\pm S D$, years & $65 \pm 12.7$ & $64.5 \pm 14.4$ & $65 \pm 5.1$ & $0.9598^{a}$ \\
\hline Sex, male/female & $46 / 44$ & $48 / 42$ & $21 / 24$ & $0.7658^{\mathrm{b}}$ \\
\hline \multicolumn{5}{|l|}{ TNM stage, $\mathrm{n}$} \\
\hline I & 22 & & & \\
\hline II & 8 & & & \\
\hline III & 13 & & & \\
\hline IV & 47 & & & \\
\hline \multicolumn{5}{|l|}{ Differentiation degree, $\mathrm{n}$} \\
\hline High & 19 & & & \\
\hline Moderate & 46 & & & \\
\hline Poor & 25 & & & \\
\hline \multicolumn{5}{|l|}{ Histological type, $\mathrm{n}$} \\
\hline Adenocarcinoma & 68 & & & \\
\hline Mucinous carcinoma & 6 & & & \\
\hline Signet ring cell carcinoma & 5 & & & \\
\hline Adenocarcinoma with signet ring cell carcinoma & 11 & & & \\
\hline \multicolumn{5}{|l|}{ Histological type, $\mathrm{n}$} \\
\hline Intestinal metaplasia & & 82 & & \\
\hline Atypical hyperplasia and other type & & 8 & & \\
\hline
\end{tabular}

${ }^{\text {aP }}$-value was calculated using one-way ANOVA. ${ }^{\mathrm{b}} \mathrm{P}-\mathrm{v}$ alue was calculated using the $\chi^{2}$ test. GC, gastric cancer; Pre, precancerous lesions; $\mathrm{NC}$, healthy control.

Previous studies have reported that miR-130b (24) is dysregulated in various cancer types, including GC (25-29). It has been reported that miR-130b promotes cell proliferation, migration and invasion, and serves as a biomarker of a poor prognosis in lung cancer (25). Zhu et al (28) reported that miR-130b is upregulated in esophageal squamous cell carcinoma tumor tissues and cells, acting as a tumor promoter by targeting SAM and SH3 domain containing 1. In ovarian cancer, miR-130b downregulation has been associated with progression, multidrug resistance and poor histological differentiation (30). In breast cancer and lung cancer, miR-130b contributes to chemoresistance by activating the phosphoinositol 3 kinase/protein kinase B and $\mathrm{Wnt} / \beta$ catenin signaling pathways $(31,32)$. Furthermore, it has been documented that miR-130b upregulation may be associated with enhanced epithelial-mesenchymal transition and angiogenesis in colorectal cancer (33). Lai et al (29) revealed that miR-130b expression is higher in gastric tissues compared with that in matched normal tissue, and this decreases the growth suppressive potential of RUNX family transcription factor 3 and contributes to tumorigenesis. Similarly, the present results demonstrated that circulating miRNA-130b was highly expressed in patients with GC, Pres and EGC, and it was significantly associated with the TNM stage of GC. Furthermore, the present study revealed that the AUC value of miR-130b was 0.887 for the diagnosis of GC, 0.911 for the diagnosis of EGC and 0.700 for the diagnosis of Pres, suggesting that miR-130b may contribute to the development of GC and could be a useful screening biomarker for EGC and Pres.
As an important parameter reflecting the heterogeneity of erythrocyte volume, RDW is often used in the diagnosis and observation of the curative effect of iron deficiency anemia, the differential diagnosis of small cell hypochromic anemia and the differential diagnosis of anemia (34). Beyazit et al (9) revealed that elevated RDW can serve as a useful biomarker for differentiating benign from malignant causes of biliary obstruction, with a sensitivity of $72 \%$ and a specificity of $69 \%$. Spell et al (10) reported that RDW yielded $84 \%$ sensitivity and $88 \%$ specificity for distinguishing between right-sided colon cancer cases and healthy subjects. In breast cancer diagnosis, Seretis et al (35) also suggested that RDW may be a novel biomarker inversely associated with the tumor grade. The present study revealed that elevated RDW in the peripheral blood was a useful biomarker for the early diagnosis of GC, which was in line with results from a previous study (8). A previous study revealed that RDW levels in GC might be influenced by inflammation and iron loss associated with chronic inflammatory status (36). An earlier study revealed that response to elevated levels of pro-inflammatory factors in cancer, erythropoietic activity, and therefore iron metabolism and homeostasis, are impaired (37). In addition, previous studies have revealed that RDW is positively associated with the conventional tumor markers CEA and CA19-9 in colorectal cancer (38), and tumor stage in ovarian cancer (39). The present study demonstrated that RDW was positively associated with circulating markers CA50 and CA125, and the pathological staging of GC. Therefore, the increased RDW may be associated with the occurrence, development and prognosis of GC, and it might be an excellent circulating marker for the early diagnosis of GC. 
Anemia is a common complication of cancer, which is associated with the energy level and quality of life score of patients (40). As a prognostic predictor, Hct has been reported in renal cell carcinoma, lung cancer and triple-negative breast cancer (41-43). Zhang et al (44) demonstrated that lower levels of $\mathrm{Hb}$ predict worse survival rate of patients with advanced GC. However, to the best of our knowledge, the value of $\mathrm{Hb}$ and Hct in the diagnosis of GC has not been reported. To the best of our knowledge, the present study was the first to reveal that both $\mathrm{Hb}$ and Hct could be useful biomarkers for GC, especially for EGC. As is well known, advanced stage is associated with a poor prognosis of GC (2). In further analysis in the present study, it was revealed that $\mathrm{Hb}$ and Hct were negatively correlated with cancer stage, which suggested that $\mathrm{Hb}$ and Hct may be associated with the severity of GC. It should be noted that numerous clinicopathological factors can affect Hct levels. For example, low Hct is more likely to occur in patients with advanced stage, anemia and low albumin (45). Therefore, these interference factors should be excluded when low Hct is used as a prognostic indicator.

PDW is a parameter reflecting the variation of platelet volume in the blood, and it is considered to be an indicator of platelet activation (46). A previous study demonstrated that PDW levels in patients with GC and Pres were lower than those in NCs (47). However, in the present study, there was no significant difference among the GC, Pre and NC groups, and the reasons for this require further study. MPV is an early indicator of platelet activation. In a study from China, Yun et al (48) stated that decreased levels of MPV in GC could be a potential biomarker for follow-up independently of tumor stage. The present study revealed higher levels of MPV in the GC and Pre groups than in the NC group, which was similar to a previous study (49). MPV/PC has been preferentially proposed as a predictor of patients with cancer (50). However, the association between MPV/PC and cancer is inconsistent. Sun et al (51) reported a higher MPV/PC ratio in patients with hepatocellular carcinoma compared with that in the control group. By contrast, a decrease in the MPV/PC ratio was detected in patients with advanced non-small cell lung carcinoma (52). In the present study, although the MPV/PC ratio of Pres was higher than that of the normal group, there was no difference between patients with GC and the control group. Additionally, the MPV/PC ratio has been recommended to be measured to differentiate iron deficiency anemia from other types of anemia (51). Therefore, the MPV/PC ratio could have potential clinical value in the identification of patients with GC. However, further investigations should be conducted to clarify the pathogenic mechanisms of the MPV/PC ratio in GC.

The other inflammatory parameters in the present study were L\#, L\%, N\#, N\%, M\#, M\%, NLR, PLR and MLR. It has been reported that platelet count, MPV, RDW, NLR and PLR can be used as circulating tumor markers to discriminate GCs from NCs (8). As reported by Chen et al (6), MLR can be used as a predictor to assess the survival rate of patients with advanced GC receiving chemotherapy. A previous study also reported that MLR may be a potential biomarker for predicting the overall survival rate of patients with advanced GC (53). The present results appear to be similar to these previous reports $(6,8)$. Studies have demonstrated that high levels of NLR and PLR are associated with a poor prognosis in colon cancer, ovarian cancer and GC $(7,8)$. The present results agree with the findings of Aldemir et al (54), Yu et al (55) and Lian et al (56). The direct mechanism is not entirely understood. However, it seems most likely that increased neutrophil- and platelet-associated inflammation and decreased lymphocyte-dependent antitumor cellular immune response are closely associated with tumorigenesis. These changes result in an increase in neutrophil and platelet levels, and a decrease in lymphocyte levels (57). In addition, the present study was one of the few studies to focus specifically on the diagnostic value of NLR and PLR in patients with GC compared with past research (58). In the present study, NLR and PLR levels in GC were higher than those in NCs, and they were independently associated with the presence of GC. Notably, the data also revealed that NLR and PLR were positively correlated with GC staging and traditional tumor markers.

Combinations of biomarkers, such as circulating miR-19a-3p and miR-483-5p, can enhance the diagnostic performance in various cancer types (59-62). Notably, Chen et al (18) recently demonstrated that miR-650 combined with CA211 could be an effective diagnostic indicator for screening of the incidence of GC. Sun et al (63) demonstrated that the combination of serum multi-dimensional biomarkers could further improve the specificity and sensitivity of GC detection, thus achieving higher diagnostic efficiency. In the present study, a predictive diagnostic model of association for GC, EGC and Pres was developed. Different combinations were tested to elucidate subsets of potential biomarkers used to separate early and advanced stage patients. Furthermore, the present study evaluated the diagnostic performance of a four-dimensional biomarker panel for GC detection using AUC analysis. Overall, the combination of four-dimensional biomarkers improved the diagnostic accuracy for GC, with an accuracy of $93.94 \%$, a DOR of 503.10 and an $\mathrm{LR}^{+}$of 41.42 , which were better than those of one-, two- or tri-dimensional biomarkers. It confirmed that such four-dimensional biomarkers had potential value as a warning sign of GC. Subsequently, the combinations of miR-130b with PDW and $\mathrm{Hb}$ had larger AUC values for EGC compared with one- or two-dimensional biomarkers, suggesting that using these combined markers may improve the early detection of GC. Finally, the present study assessed the diagnostic performance of a three-dimensional biomarker panel for Pre detection using AUC analysis. Results demonstrated that the combination of miR-130b and PDW was better than any other single indicator or combination of indicators. Based on the aforementioned analysis results, it was hypothesized that circulating miR-130b and blood routine parameters were effective early warning indicators for early diagnosis of GC.

Although the results of the present study have certain clinical value, there were several limitations. Firstly, in view of the small number of included cases, although valuable results have been achieved in the present study, the time point at which these indicators are measured is unclear and their specific application has not been assessed. Therefore, clear consequences cannot be obtained, and further research is required. Secondly, Helicobacter pylori is an important carcinogen of GC. The present study did not investigate the relationship between $H$. pylori and mir-130 in GC, which is also a focus of further research. Finally, there was no specific 
genotyping in patients with GC, and miRNA expression might be different between gene subtypes. Therefore, the significance of miR-130b and blood routine parameters in the early diagnosis of $\mathrm{GC}$ requires further study.

In conclusion, miRNA-130b, which was upregulated in GC, may be a novel early diagnostic marker for GC. Furthermore, using ROC curve analysis, the optimal cut-off values of the markers could be determined and the diagnosis of GC could be improved based on these blood routine markers. Novel screening models (miR-130b combined with blood routine parameters) were developed in the present study, and the optimal application of these circulating tumor markers could promote the clinical screening and diagnosis of GC, which patients may be more willing to accept for the detection of GC over more invasive tests.

\section{Acknowledgements}

Not applicable.

\section{Funding}

The present study was supported by a grant from the Guangxi Zhuang Autonomous Region Health and Family Planning Commission (no. Z2014613). The funders had no role in study design, data collection and analysis, decision to publish, or preparation of the manuscript.

\section{Availability of data and materials}

The datasets used and/or analyzed during the current study are available from the corresponding author on reasonable request.

\section{Authors' contributions}

JC conceived the idea, designed the study protocol and performed the experiments. ZL, GG, YM, HZ, WH, LW, XH, JD, CL, HL, JF and YS carried out the data collection and data analysis, and provided the critical revision. XG analyzed and interpreted the data, was involved in drafting and revision of the manuscript and gave final approval of the version to be published. JC, ZL and YS confirm the authenticity of all the raw data. All authors read and approved the final manuscript, and agree to be accountable for all aspects of the research in ensuring that the accuracy or integrity of any part of the work are appropriately investigated and resolved.

\section{Ethics approval and consent to participate}

The present study was approved by The Ethical Review Committee of Affiliated Liutie Central Hospital of Guangxi Medical University (Liuzhou, China). All patients provided written informed consent.

\section{Patient consent for publication}

Not applicable.

\section{Competing interests}

The authors declare that they have no competing interests.

\section{References}

1. Zhang H, Zhong X, Zhang X, Shang D, Zhou YI and Zhang C: Enhanced anticancer effect of ABT-737 in combination with naringenin on gastric cancer cells. Exp Ther Med 11: 669-673, 2016.

2. Hamashima C: The burden of gastric cancer. Ann Transl Med 8: $734,2020$.

3. Feng F, Tian Y, Xu G, Liu Z, Liu S, Zheng G, Guo M, Lian X, Fan D and Zhang H: Diagnostic and prognostic value of CEA, CA19-9, AFP and CA125 for early gastric cancer. BMC Cancer 17: 737, 2017.

4. Thanh Huong P, Gurshaney S, Thanh Binh N, Gia Pham A, Hoang Nguyen H, Thanh Nguyen X, Pham-The H, Tran PT, Truong Vu K, Xuan Duong N, et al: Emerging role of circulating tumor cells in gastric cancer. Cancers (Basel) 12: 695, 2020.

5. Hussain SP and Harris CC: Inflammation and cancer: An ancient link with novel potentials. Int J Cancer 121: 2373-2380, 2007.

6. Chen L, Hao Y, Zhu L, Li S, Zuo Y, Zhang Y, Song H and Xue Y: Monocyte to lymphocyte ratio predicts survival in patients with advanced gastric cancer undergoing neoadjuvant chemotherapy. Onco Targets Ther 10: 4007-4016, 2017.

7. Jiang Y, Xu H, Jiang H, Ding S and Zheng T: Pretreatment neutrophil-lymphocyte count ratio may associate with gastric cancer presence. Cancer Biomark 16: 523-528, 2016.

8. Pietrzyk L,PlewaZ, Denisow-Pietrzyk M,Zebrowski R and Torres K: Diagnostic power of blood parameters as screening markers in gastric cancer patients. Asian Pac J Cancer Prev 17: 4433-4437, 2016.

9. Beyazit Y, Kekilli M, Ibis M, Kurt M, Sayilir A, Onal IK, Purnak T, Oztas E, Tas A, Yesil Y and Arhan M: Can red cell distribution width help to discriminate benign from malignant biliary obstruction? A retrospective single center analysis. Hepatogastroenterology 59: 1469-1473, 2012.

10. Spell DW, Jones DV Jr, Harper WF and David Bessman J: The value of a complete blood count in predicting cancer of the colon. Cancer Detect Prev 28: 37-42, 2004.

11. Dhahbi JM, Atamna H, Li R, Yamakawa A, Guerrero N, Lam HT, Mote P and Spindler SR: MicroRNAs circulate in the hemolymph of drosophila and accumulate relative to tissue microRNAs in an Age-dependent manner. Genomics Insights 9: 29-39, 2016.

12. Schetter AJ, Heegaard NH and Harris CC: Inflammation and cancer: Interweaving microRNA, free radical, cytokine and p53 pathways. Carcinogenesis 31: 37-49, 2010.

13. Garrido-Cano I, Constâncio V, Adam-Artigues A, Lameirinhas A, Simón S, Ortega B, Martínez MT, Hernando C, Bermejo B, Lluch A, et al: Circulating miR-99a-5p expression in plasma: A potential biomarker for early diagnosis of breast cancer. Int J Mol Sci 21: 7427, 2020.

14. Ghosh S, Bhowmik S, Majumdar S, Goswami A, Chakraborty J, Gupta S, Aggarwal S, Ray S, Chatterjee R, Bhattacharyya S, et al: The exosome encapsulated microRNAs as circulating diagnostic marker for hepatocellular carcinoma with low alpha fetoprotein. Int J Cancer 147: 2934-2947, 2020.

15. Ying L, Du L, Zou R, Shi L, Zhang N, Jin J, Xu C, Zhang F, Zhu C, Wu J, et al: Development of a serum miRNA panel for detection of early stage non-small cell lung cancer. Proc Natl Acad Sci USA 117: 25036-25042, 2020.

16. Liu $X$ and Chu KM: Exosomal miRNAs as circulating biomarkers for prediction of development of haematogenous metastasis after surgery for stage II/III gastric cancer. J Cell Mol Med 24: 6220-6232, 2020.

17. Kong Y, Ning L, Qiu F, Yu Q and Cao B: Clinical significance of serum miR-25 as a diagnostic and prognostic biomarker in human gastric cancer. Cancer Biomark 24: 477-483, 2019.

18. Chen J, Wu L, Sun Y, Luo C, Chen X, Wu L, Ding J, Pan G, Han C, Wu Z and Shen Y: Diagnostic value and clinical significance of circulating miR-650 and CA211 in detecting of gastric carcinoma. Oncol Lett 20: 254, 2020.

19. Catalano V,SistiV,SpadaD,GrazianoF,CicettiM,AlessandroniPGP, Baldelli AM, Casadei V, Fedeli SL, Rossi D, et al: Assessment of the 7th edition of the AJCC classification and a proposal of a new classification in patients with gastric cancer undergoing D2 gastrectomy. J Clin Oncol 30 (Suppl 15): S4084, 2012.

20. Bosman FT, Carneiro F, Hruban RH and Theise ND (eds): WHO Classification of Tumours of the Digestive System. In: World Health Organization Classification of Tumours. Volume 3. 4th edition, Lyon France, 2010.

21. Yamano T, Kubo S, Sonoda E, Kominato T, Kimura K, Yasuhara M, Kataoka K, Son J, Babaya A, Takenaka Y, et al: Assessment of circulating microRNA specific for patients with familial adenomatous polyposis. PLoS One 16: e0250072, 2021. 
22. Livak KJ and Schmittgen TD: Analysis of relative gene expression data using real-time quantitative PCR and the 2(-Delta Delta C(T)) Method. Methods 25: 402-408, 2001.

23. Chen J, Wu L, Sun Y, Yin Q, Chen X, Liang S, Meng Q, Long H, Li F, Luo C and Xiao X: Mir-421 in plasma as a potential diagnostic biomarker for precancerous gastric lesions and early gastric cancer. PeerJ 7: e7002, 2019.

24. Burmistrova OA, Goltsov AY, Abramova LI, Kaleda VG, Orlova VA and Rogaev EI: MicroRNA in schizophrenia: Genetic and expression analysis of miR-130b (22q11). Biochemistry (Mosc) 72: 578-582, 2007.

25. Kim Y, Kim H, Bang S, Jee S and Jang K: MicroRNA-130b functions as an oncogene and is a predictive marker of poor prognosis in lung adenocarcinoma. Lab Invest 101: 155-164, 2021.

26. Ou C, Peng NF, Li H, Peng YC and Li LJQ: The potential mechanism of miR-130b on promotion of the invasion and metastasis of hepatocellular carcinoma by inhibiting Notch-Dll1. J Recept Signal Transduct Res 40: 157-165, 2020.

27. Hashimoto Y, Shiina M, Dasgupta P, Kulkarni P, Kato T, Wong RK, Tanaka Y, Shahryari V, Maekawa S, Yamamura S, et al: Upregulation of miR-130b contributes to risk of poor prognosis and racial disparity in African-American prostate cancer. Cancer Prev Res (Phila) 12: 585-598, 2019.

28. Zhu Y, Ma Y, Peng H, Gong L, Xiao M, Xiang L, He D and Cao K: miR-130b promotes the progression of oesophageal squamous cell carcinoma by targeting SASH1. J Cell Mol Med 23: 93-103, 2019.

29. Lai KW, Koh KX, Loh M, Tada K, Subramaniam MM, Lim XY, Vaithilingam A, Salto-Tellez M, Iacopetta B, Ito Y, et al: MicroRNA-130b regulates the tumour suppressor RUNX3 in gastric cancer. Eur J Cancer 46: 1456-1463, 2010.

30. Yang C, Cai J, Wang Q, Tang H, Cao J, Wu L and Wang Z: Epigenetic silencing of miR-130b in ovarian cancer promotes the development of multidrug resistance by targeting colony-stimulating factor 1. Gynecol Oncol 124: 325-334, 2012.

31. Miao Y, Zheng W, Li N, Su Z, Zhao L, Zhou H and Jia L: MicroRNA-130b targets PTEN to mediate drug resistance and proliferation of breast cancer cells via the PI3K/Akt signaling pathway. Sci Rep 7: 41942, 2017.

32. Zhang Q, Zhang B, Sun L, Yan Q, Zhang Y, Zhang Z, Su Y and Wang C: MicroRNA-130b targets PTEN to induce resistance to cisplatin in lung cancer cells by activating Wnt/ $\beta$-catenin pathway. Cell Biochem Funct 36: 194-202, 2018.

33. Colangelo T, Fucci A, Votino C, Sabatino L, Pancione M,Laudanna C, Binaschi M, Bigioni M, Maggi CA,Parente D, et al: MicroRNA-130b promotes tumor development and is associated with poor prognosis in colorectal cancer. Neoplasia 15: 1086-1099, 2013.

34. Zhao L, Mao ZG, Jiang H, Qin L, Huang CY and Tan B: Value of MCV/RDW combined with reticulocyte parameters in differential diagnosis of Anemia diseases. Zhongguo Shi Yan Xue Ye Xue Za Zhi 23: 1662-1666, 2015 (In Chinese).

35. Seretis C,Seretis F,Lagoudianakis E, Gemenetzis G and Salemis NS Is red cell distribution width a novel biomarker of breast cancer activity? Data from a pilot study. J Clin Med Res 5: 121-126, 2013.

36. Sategna Guidetti C, Scaglione N and Martini S: Red cell distribution width as a marker of coeliac disease: A prospective study. Eur J Gastroenterol Hepatol 14: 177-181, 2002.

37. Lippi G, Targher G, Montagnana M, Salvagno GL, Zoppini G and Guidi GC: Relation between red blood cell distribution width and inflammatory biomarkers in a large cohort of unselected outpatients. Arch Pathol Lab Med 133: 628-632, 2009

38. Li Y, Xing C, Wei M, Wu H, Hu X, Li S, Sun G, Zhang G, Wu B, Zhang F and Li Z: Combining red blood cell distribution width (RDW-CV) and CEA predict poor prognosis for survival outcomes in colorectal cancer. J Cancer 10: 1162-1170, 2019.

39. Qin YY, Wu YY, Xian XY, Qin JQ, Lai ZF, Liao L and Lin FQ: Single and combined use of red cell distribution width, mean platelet volume, and cancer antigen 125 for differential diagnosis of ovarian cancer and benign ovarian tumors. J Ovarian Res 11: 10, 2018

40. Gilreath JA and Rodgers GM: How I treat cancer-associated anemia. Blood 136: 801-813, 2020

41. Zhang X, Zhang F, Qiao W, Zhang X, Zhao Z and Li M: Low hematocrit is a strong predictor of poor prognosis in lung cancer patients. Biomed Res Int 2018: 6804938, 2018.

42. Seda CJ, Salas AS, Sánchez CG, Blasco JM, García IO, Sánchez JM, Ruíz CB, Navarro SM and López RA: Thrombocytosis and hematocrit as prognostic factors in renal carcinoma. Arch Esp Urol 64: 883-890, 2011.

43. Chen B, Dai D, Tang H, Ai X, Chen X, Zhang X, Li Z and Xie X: Pretreatment hematocrit is superior to hemoglobin as a prognostic factor for triple negative breast cancer. PLoS One 11: e0165133, 2016.
44. Zhang S, Lu M, Li Y, Li J and Shen LJCo: A lower haemoglobin level predicts a worse survival of patients with advanced gastric cancer. Clin Oncol (R Coll Radiol) 26: 239-240, 2014.

45. Chen J, Li Y and Cui H: Preoperative low hematocrit is an adverse prognostic biomarker in ovarian cancer. Arch Gynecol Obstet 303: 767-775, 2021

46. Liu F, Hu HJ, Regmi P, Jin YW, Ma WJ, Wang JK, Zou RQ and Li FY: Elevated platelet distribution width predicts poor prognosis in gallbladder carcinoma. Cancer Manag Res 13: 4647-4655, 2021.

47. Aksoy EK, Kantarcı S, Torgutalp M, Akpınar MY, Sapmaz FP, Yalçın GŞ, Uzman M, Şimşek GG and Nazlıgül Y: The importance of complete blood count parameters in the screening of gastric cancer. Prz Gastroenterol 14: 183-187, 2019.

48. Yun ZY, Li N, Zhang X, Zhang H, Bu Y, Sun Y, Liu T, Wang RT and $\mathrm{Yu}$ KJ: Mean platelet volume, platelet distribution width and carcinoembryonic antigen to discriminate gastric cancer from gastric ulcer. Oncotarget 8: 62600-62605, 2017.

49. Kılınçalp S, Ekiz F, Başar O, Ayte MR, Coban S, Yılmaz B, Altınbaş A, Başar N, Aktaş B, Tuna Y and.Erbiş H: Mean platelet volume could be possible biomarker in early diagnosis and monitoring of gastric cancer. Platelets 25: 592-594, 2014.

50. Tuncel T, Ozgun A,Emirzeoglu L, Celik S, Bilgi O and Karagoz B: Mean platelet volume as a prognostic marker in metastatic colorectal cancer patients treated with bevacizumab-combined chemotherapy. Asian Pac J Cancer Prev 15: 6421-6423, 2014.

51. Sun YC, Yang JJ, Suh JT, Lee WI, Lee HJ and Park TS: Mean platelet volume/platelet count ratio in anemia. Platelets 24: 244-245, 2013.

52. Inagaki N, Kibata $\mathrm{K}$, Tamaki $\mathrm{T}$, Shimizu $\mathrm{T}$ and Nomura $\mathrm{S}$ : Prognostic impact of the mean platelet volume/platelet count ratio in terms of survival in advanced non-small cell lung cancer. Lung Cancer 83: 97-101, 2014.

53. Song S, Li C, Li S, Gao H, Lan X and Xue Y: Derived neutrophil to lymphocyte ratio and monocyte to lymphocyte ratio may be better biomarkers for predicting overall survival of patients with advanced gastric cancer. Onco Targets Ther 10: 3145-3154, 2017.

54. Aldemir MN, Turkeli M, Simsek M, Yildirim N, Bilen Y, Yetimoglu H, Bilici M and Tekin SB: Prognostic value of baseline neutrophil-lymphocyte and platelet-lymphocyte ratios in local and advanced gastric cancer patients. Asian Pac J Cancer Prev 16: 5933-5937, 2015.

55. Yu L, Lv CY, Yuan AH, Chen W and Wu AW: Significance of the preoperative neutrophil-to-lymphocyte ratio in the prognosis of patients with gastric cancer. World J Gastroenterol 21: 6280-6286, 2015

56. Lian L, Xia YY, Zhou C, Shen XM, Li XL, Han SG, Zheng Y, MaoZQ, Gong FR, Wu MY, et al: Application of platelet/lymphocyte and neutrophil/lymphocyte ratios in early diagnosis and prognostic prediction in patients with resectable gastric cancer. Cancer Biomark 15: 899-907, 2015.

57. Hsu JT, Liao CK, Le PH, Chen TH, Lin CJ, Chen JS, Chiang KC and Yeh TS: Prognostic value of the preoperative neutrophil to lymphocyte ratio in resectable gastric cancer. Medicine (Baltimore) 94: e1589, 2015

58. Deng Q, He B, Liu X, Yue J, Ying H, Pan Y, Sun H, Chen J, Wang F, Gao T, et al: Prognostic value of pre-operative inflammatory response biomarkers in gastric cancer patients and the construction of a predictive model. J Transl Med 13: 66, 2015

59. Yang SH, Wang XL, Cai J and Wang SH: Diagnostic value of circulating PIGF in combination with Flt-1 in early cervical cancer. Curr Med Sci 40: 973-978, 2020.

60. Leandersson P, Åkesson A, Hedenfalk I, Malander S and Borgfeldt C: A multiplex biomarker assay improves the diagnostic performance of HE4 and CA125 in ovarian tumor patients. PLoS One 15: e0240418, 2020.

61. Huang J, Zheng Y, Xiao X, Liu C, Lin J, Zheng S, Yang B and Ou Q: A circulating long noncoding RNA panel serves as a diagnostic marker for hepatocellular carcinoma. Dis Markers 2020: 5417598, 2020.

62. Cheng J, Yang A, Cheng S, Feng L, Wu X, Lu X, Zu M, Cui J, Yu H and Zou L: Circulating miR-19a-3p and miR-483-5p as novel diagnostic biomarkers for the early diagnosis of gastric cancer. Med Sci Monit 26: e923444, 2020.

63. Sun L, Tu H, Chen T, Yuan Q, Liu J, Dong N and Yuan Y: Three-dimensional combined biomarkers assay could improve diagnostic accuracy for gastric cancer. Sci Rep 7: 11621, 2017.

This work is licensed under a Creative Commons Attribution-NonCommercial-NoDerivatives 4.0 International (CC BY-NC-ND 4.0) License. 